\title{
RADIAL VARIATION OF ATTENUATION AND STAR FORMATION IN THE LARGEST LATE-TYPE DISKS OBSERVED WITH GALEX
}

\author{
Samuel Boissier, ${ }^{1,2}$ Armando Gil de Paz, ${ }^{1,3}$ Alessandro Boselli, ${ }^{2}$ Barry F. Madore, ${ }^{1,4}$ Véronique Buat, ${ }^{2}$ \\ Luca Cortese, ${ }^{2}$ Denis Burgarella, ${ }^{2}$ Juan Carlos Muñoz Mateos, ${ }^{3}$ Tom A. Barlow, ${ }^{5}$ Karl Forster, ${ }^{5}$ \\ Peter G. Friedman, ${ }^{5}$ D. Christopher Martin, ${ }^{5}$ Patrick Morrissey, ${ }^{5}$ Susan G. Neff, ${ }^{6}$ \\ David Schiminovich, ${ }^{7}$ Mark Seibert, ${ }^{5}$ Todd Small, ${ }^{5}$ Ted K. Wyder, ${ }^{5}$ \\ Luciana Bianchi, ${ }^{8}$ José Donas, ${ }^{2}$ Timothy M. Heckman, ${ }^{9}$ Young-Wook Lee, ${ }^{10}$ \\ Bruno Milliard, ${ }^{2}$ R. Michael Rich, ${ }^{11}$ Alex S. Szalay, 9 \\ Barry Y. Welsh, ${ }^{12}$ and Sukyoung K. YI ${ }^{10}$ \\ Received 2006 April 20; accepted 2006 July 28
}

\begin{abstract}
For a sample of 43 nearby, late-type galaxies, we have investigated the radial variation of both the current star formation rate and the dust-induced UV light attenuation. To do this we have cross-correlated IRAS images and GALEX observations for each of these galaxies and compiled observations of the gas ( $\mathrm{CO}$ and $\mathrm{H} \mathrm{I})$ and metal-abundance gradients found in the literature. We find that attenuation correlates with metallicity. We then use the UV profiles, corrected for attenuation, to study several variants of the Schmidt law and conclude that our results are compatible with a simple law similar to the one of Kennicutt extending smoothly to lower surface densities, but with considerable scatter. We do not detect an abrupt break in the UV light at the threshold radius derived from $\mathrm{H} \alpha$ data (at which the $\mathrm{H} \alpha$ profile shows a break and beyond which only a few $\mathrm{H}$ II regions are usually found). We interpret the $\mathrm{H} \alpha$ sudden break not as a change in the star formation regime (as often suggested), but as the vanishingly small number of ionizing stars corresponding to low levels of star formation.
\end{abstract}

Subject headings: dust, extinction — galaxies: spiral — infrared: galaxies — ultraviolet: galaxies

Online material: color figures, extended figure set

\section{INTRODUCTION}

Interstellar dust affects our view of galaxies from the UV to the near-infrared. High-redshift galaxies are commonly studied in the rest-frame UV where dust effects can be extremely severe, and our estimation of the "cosmic" star formation rate (SFR) (e.g., Schiminovich et al. 2005) crucially depends on the corrections applied to account for the dust attenuation of starlight.

Radiative transfer models suggest that the ratio of far-infrared to UV radiation is a reliable measure of the UV attenuation (Buat \& Xu 1996; Witt \& Gordon 2000; Panuzzo et al. 2003), depending weakly on the geometry of stars and dust, the extinction law,

\footnotetext{
1 Observatories of the Carnegie Institution of Washington, 813 Santa Barbara Street, Pasadena, CA 91101; boissier@ociw.edu, agpaz@ociw.edu, madore@ociw .edu.

${ }^{2}$ Laboratoire d'Astrophysique de Marseille, BP 8, Traverse du Siphon, 13376 Marseille Cedex 12, France.

3 Departamento de Astrofísica y CC. de la Atmósfera, Universidad Complutense de Madrid, Avda. de la Complutense, s/n, E-28040 Madrid, Spain.

4 Infrared Processing and Analysis Center California Institute of Technology Mail Code 100-22 770 South Wilson Avenue, Pasadena, CA 91125.

5 California Institute of Technology, MC 405-47, 1200 East California Boulevard, Pasadena, CA 91125.

${ }^{6}$ Laboratory for Astronomy and Solar Physics, NASA Goddard Space Flight Center, Greenbelt, MD 20771.

7 Department of Astronomy, Columbia University, New York, NY 10027.

8 Center for Astrophysical Sciences, The Johns Hopkins University, 3400 North Charles Street, Baltimore, MD 21218.

9 Department of Physics and Astronomy, The Johns Hopkins University, Homewood Campus, Baltimore, MD 21218.

${ }^{10}$ Center for Space Astrophysics, Yonsei University, Seoul 120-749, Korea.

11 Department of Physics and Astronomy, University of California, Los Angeles, CA 90095.

12 Space Sciences Laboratory, University of California at Berkeley, 601
} Campbell Hall, Berkeley, CA 94720. or the nature of the underlying stellar population. Since this ratio is not always available, the slope of the UV spectrum reddening with respect to local starbursts (Calzetti et al. 1994; Meurer et al. $1995,1999)$ has been commonly used as a metric to estimate the amount of attenuation. This allows one to compare the attenuation (as measured by the far-infrared/UV ratio) to the slope of the UV spectrum in a diagnostic plot, the commonly called "IRX- $\beta$ relationship." Several recent works, mostly based on GALEX observations, cast doubt on the IRX- $\beta$ relation that has been most commonly used in recent years (Buat et al. 2005; Cortese et al. 2006; Seibert et al. 2005; Gil de Paz et al. 2007). Independent studies have come to similar conclusions, e.g., Bell (2002) for normal spirals and Bell et al. (2002) for individual regions in the LMC.

These works suggests either that there is no relationship (or a very noisy one) or that a relationship exists but differs from the starburst one, depending on the selection of the sample (infrared vs. UV or optically selected).

The largest angular sized galaxies in the GALEX Atlas of Nearby Galaxies (Gil de Paz et al. 2007) allow us to revisit the IRX- $\beta$ relationship. Indeed, several tens of nearby galaxies observed by GALEX are large enough to have been resolved even by IRAS. The combination of the UV data of GALEX and infrared of IRAS opens up the possibility of looking at the spatially resolved IRX- $\beta$ relationship across a relatively large sample of galaxies. Even if the number of resolved objects in this study is smaller than the total number of objects used in the global studies, each of our galaxies provides a number of independent points along the radial profile. Regions situated at the same radius within a galaxy are relatively homogeneous (for instance similar metallicities), and thus radial profiles are physically relevant. 
Another compelling reason to study attenuation ${ }^{13}$ in these galaxies is that, as our closest neighbors, they have been extensively surveyed at other wavelengths, and in the published literature there are metallicity gradient and gas density profiles, etc., that allow us to go one step further and try to characterize how the properties of dust might depend on these other physical quantities. We also produce star formation rate radial profiles derived from the UV (and not $\mathrm{H} \alpha$ data), corrected for the dust attenuation for all our galaxies. These profiles can be used to study how the star formation rate depends on the gas density, i.e., the so-called Schmidt law. We will be able to use these star formation rate profiles to finally study the threshold radius beyond which little (if any) star formation is observed. Many studies suggest that the threshold radius corresponds to the point where gas density becomes lower than a critical density for star formation (e.g., Martin \& Kennicutt 2001). It is important to bring as many constraints as possible to bear on the complex problem of star formation and this paper brings its share, by using UV GALEX data. Indeed, while many other studies of the Schmidt law (Kennicutt 1998a; Boissier et al. 2003; Wong \& Blitz 2002) are based on $\mathrm{H} \alpha$ data, the star formation rate in this paper is derived from the UV, which "averages" the star formation on longer timescales than $\mathrm{H} \alpha$.

Recombination lines as $\mathrm{H} \alpha$ are emitted only in the presence of a large ionizing flux, coming mostly from massive stars $(M>$ $\left.10 M_{\odot}\right)$ with lifetimes shorter than $2 \times 10^{7} \mathrm{yr}$. On the contrary, the UV continuum is emitted by stars with lifetimes up to $10^{8} \mathrm{yr}$ (a review of the various star formation indicators and their associated lifetimes is given in Kennicutt 1998b). Because of the scarcity of UV data before GALEX, little work had been done in the UV on the star formation law. Some exceptions are that of Buat et al. (1989) and Bersier et al. (1994) concerning, respectively, M81 and M51, or Buat (1989) and Donas et al. (1987) for studies using sample of nearby galaxies, but these papers looked only at the integrated properties of galaxies and employed rather simple recipes for dust correction. The radial variation of the UV-derived star formation rate, correctly dust-corrected via the far-infraredto-UV ratio, is the subject of this paper. This is an important work as we now know that the UV also shows us low levels of star formation (XUV galaxies, e.g., Gil de Paz et al. 2005; Thilker et al. $2005,2007)$, in regions with such low dust content that the farinfrared emission is undetected by IRAS, and that the UV is likely to be weakly affected by extinction.

In $\S 2$, we discuss how the data sets were assembled and prepared in order to undertake this study of the radial variation of the attenuation, of the star formation, and of their relation to other physical quantities in those same galaxies. In $\S 3$, we present our study of the attenuation derived from these data, and the consequences concerning the resulting star formation law are presented in $\S 4$.

\section{PROFILES: DATA AND PROCEDURE}

Our initial sample consists of 48 late-type galaxies ( RC3 T type going from 0 to +10 , thus including all spirals from $\mathrm{S} 0 /$ a to $\mathrm{Sm}$ and Magellanic irregulars Im) with UV data in the GALEX Atlas of Nearby Galaxies (Gil de Paz et al. 2007), larger than $8^{\prime}$ (as measured by $D_{25}$ diameter corresponding to the $B$-band isophote of $25 \mathrm{mag} \operatorname{arcsec}^{-2}$ ). This size allows for spatial resolution of these galaxies at all the wavelengths needed, including the 60 and $100 \mu \mathrm{m}$ IRAS data.

\footnotetext{
13 "Attenuation" is the loss of flux due to the presence of dust. It is sometimes considered that "extinction" should be used only for the dust properties and not the global effect of dust on the galaxy light, also depending on, e.g., the geometry of the system. "Extinction" is nevertheless often used with the same meaning as "attenuation."
}

All the IRAS images were obtained using HIRES requests to the IPAC Web page. ${ }^{14}$ The method used in HIRES is described in Aumann et al. (1990); examples of images obtained with HIRES have been presented by Rice (1993) for a sample of nearby galaxies (the HIRES procedure failed to converge for NGC 3109 , and this galaxy was removed from our analysis). Because of the IRAS aperture itself and the complex pattern of observations, the resulting HIRES images can be quite asymmetric. HIRES produces simulated images of point sources distributed into the image at the sky position of the targeted object. The first step in our procedure was to apply an asymmetric Gaussian convolution in order to produce a circular image at 60 and $100 \mu \mathrm{m}$ of these point sources and to apply the same convolution to the HIRES reconstructed image of the galaxy. In the UV, the GALEX far- and near-ultraviolet (FUV and NUV, respectively, around 1516 and $2267 \AA$ ) images of the Atlas (Gil de Paz et al. 2007) were used. For four galaxies, no FUV data were available (NGC 1365, NGC 3628, M98, M106), and they are excluded from our analysis, which in the end includes 43 galaxies. Given this selection process, this sample is not complete in any sense, although it should be representative of optically selected galaxies (a comparison of the GALEX Atlas of Nearby Galaxies and the Nearby Field Galaxy Survey of Jansen et al. 2000 is given in Gil de Paz et al. 2007).

Stars were removed using the star masks of the Atlas and then interpolated over. Foreground Galactic extinction was corrected as in Gil de Paz et al. (2007): using the values of the color excess $E(B-V)$ given by the maps of Schlegel et al. (1998) and a Galactic extinction curve. Images in FUV, NUV, and at $60 \mu \mathrm{m}$ were then convolved with an elliptical Gaussian function in order to match the resolution to the one measured in the HIRES simulation of point sources at $100 \mu \mathrm{m}$ (after the asymmetric Gaussian convolution discussed above). The spatial resolution of IRAS is relatively poor. However, this might help us to avoid difficulties in interpreting the UV/IR balance since the dust of small regions could be heated by the UV emission from neighboring regions.

Profiles were finally computed within elliptical annuli with ELLIPSE in IRAF $^{15}$ using convolved images, with fixed center, ellipticity, and position angles (as given in Gil de Paz et al. 2007 and Table 1). The step in major-axis radius between isophotes was chosen to be the measured resolution (close to $1.5^{\prime}$ on average and given in Table 1).

The infrared profiles were combined as in Dale et al. (2001) in order to compute a "far-infrared" profile $\left(F_{\mathrm{FIR}}\right.$ in $\left.\mathrm{W} \mathrm{m}^{-2} \operatorname{arcsec}^{-2}\right)$ and a "total-infrared" profile $\left(F_{\mathrm{TIR}}\right)$, as described below:

$$
F_{\mathrm{FIR}}=1.26 \times 10^{-14}\left(2.58 f_{60}+f_{100}\right)
$$

( $f_{\lambda}$ are the IRAS flux surface densities in $\mathrm{Jy} \operatorname{arcsec}^{-2}$ ) and

$$
\begin{aligned}
\log \left(F_{\mathrm{TIR}}\right)= & \log \left(F_{\mathrm{FIR}}\right)+0.2738-0.0282 r+0.7281 r^{2} \\
& +0.6208 r^{3}+0.9118 r^{4}
\end{aligned}
$$

where $r=\log \left(f_{60} / f_{100}\right)$

Attenuation profiles (expressed in magnitude) were then computed using the fit of Buat et al. (2005)

$$
\begin{aligned}
A(\mathrm{FUV})= & -0.0333 X^{3}+0.3522 X^{2} \\
& +1.1960 X+0.4967
\end{aligned}
$$

\footnotetext{
14 See http://irsa.ipac.caltech.edu/IRASdocs/hires_over.html.

15 IRAF is distributed by the National Optical Astronomy Observatories, which are operated by the Association of Universities for Research in Astronomy, Inc., under cooperative agreement with the National Science Foundation.
} 
TABLE 1

Sample: Basic Parameters Used and References

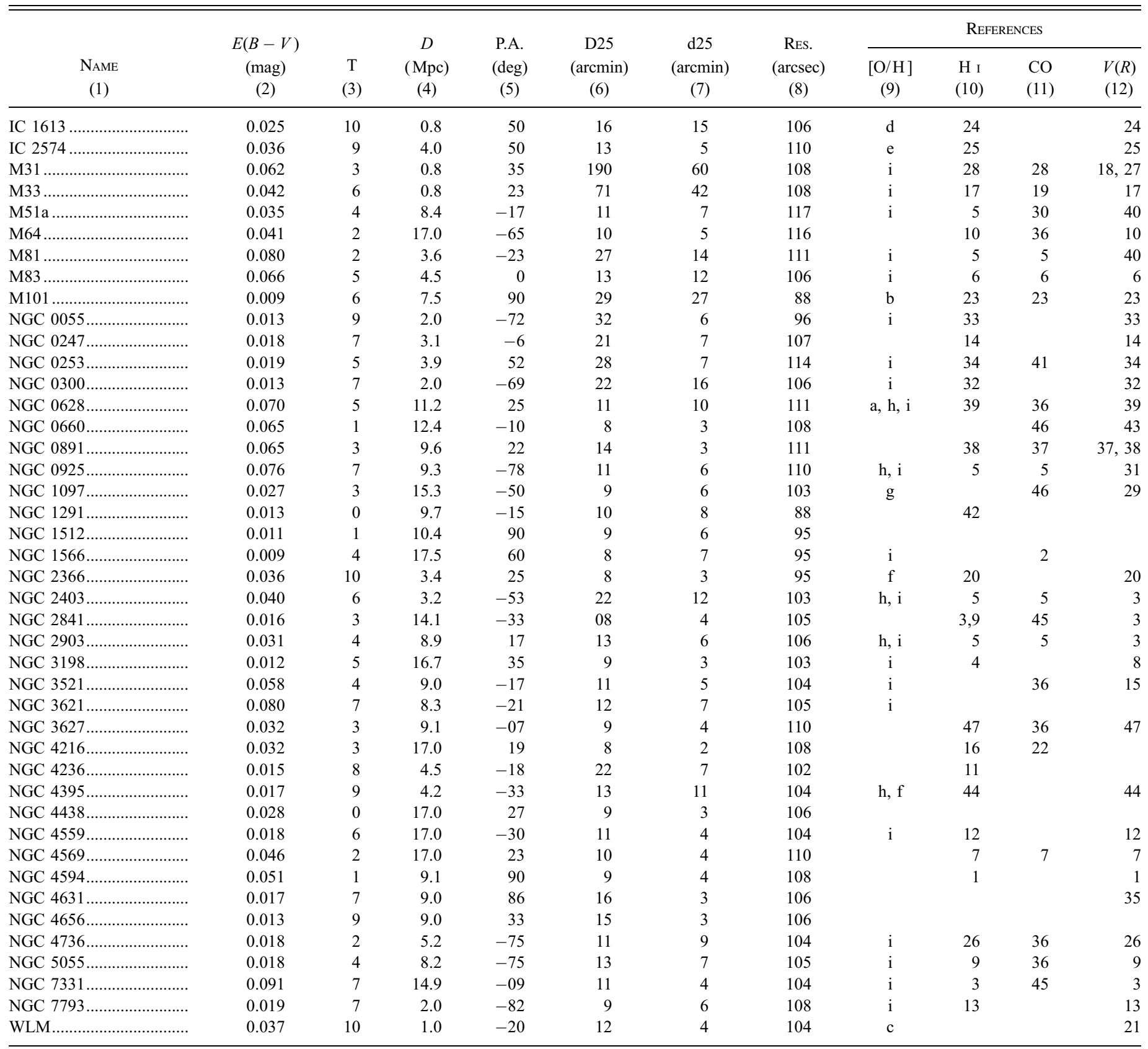

Notes.-The columns give (1) object name, (2) Galactic color excess ( Schlegel et al. 1998), (3) RC3 morphological type, (4) distance (Mpc) taken from the GALEX Atlas of Nearby Galaxies (Gil de Paz et al. 2007), (5) position angle (degree), (6) D25: major-axis diameter (minutes), (7) d25: minor-axis diameter (minutes), (8) FWHM of the restored $100 \mu \mathrm{m}$ beam in arcsec (see $\S 2$ for details), (9) references for $\mathrm{O} / \mathrm{H}$ gradients, $(10-12)$ references for $\mathrm{H}$, $\mathrm{CO}$, and rotation curve data.

References.-References for abundance gradients [O/H]: (a) Belley \& Roy 1992; (b) Kennicutt et al. 2003a; (c) Lee et al. 2003a; (d) Lee et al. 2003b; (e) Masegosa et al. 1991; (f) Roy et al. 1996; (g) Storchi-Bergmann et al. 1996; (h) van Zee et al. 1998; (i) Zaritsky et al. 1994.

References for gas [H I, CO] and kinematic [V(R)] data: (1) Bajaja et al. 1984; (2) Bajaja et al. 1995; (3) Begeman (1987; (4) Begeman 1989; (5) Boissier et al. 2003 and references therein; (6) Boissier et al. 2005 and references therein ; (7) Boselli et al. 2006 and references therein; (8) Bosma 1978; (9) Bosma 1981; (10) Braun et al. 1994; (11) Braun 1997; (12) Broeils \& van Woerden 1994; (13) Carignan \& Puche 1990a; (14) Carignan \& Puche 1990b); (15) Casertano \& van Gorkom 1991; (16) Cayatte et al. 1994; (17) Corbelli 2003; (18) Dame et al. 1993; (19) Heyer et al. 2004; (20) Hunter et al. 2001; (21) Jackson et al. 2004; (22) Kenney \& Young 1988; (23) Kenney et al. 1991; (24) Lake \& Skillman 1989; (25) Martimbeau et al. 1994; (26) Mulder \& van Driel 1993; (27) Newton \& Emerson 1977; (28) Nieten et al. 2006; (39) Ondrechen et al. 1989; (30) Paglione et al. 2001; (31) Pisano et al. 1998; (32) Puche et al. 1990; (33) Puche et al. 1991b; (34) Puche et al. 1991a; (35) Rand 1994; (36) Regan et al. 2001; (37) Sakamoto et al. 1997; (38) Sancisi \& Allen 1979; (39) Shostak \& van der Kruit 1984; (40) Sofue 1997; (43) Sorai et al. 2000; (44) van Driel et al. 1988; (45) van Driel et al. 1995; (46) Wevers et al. 1986; (48) Young \& Scoville 1982; (50) Young et al. 1995; (51) Zhang et al. 1993.

where $X=\log \left(F_{\mathrm{TIR}} / F_{\mathrm{FUV}}\right), F_{\mathrm{FUV}}=\nu_{\text {fuv }} f_{\nu_{\text {fuv }}}$, and $f_{\nu_{\text {fuv }}}$ is the FUV flux surface density in $\mathrm{W} \mathrm{m}^{-2} \mathrm{~Hz}^{-1}$ arcsec ${ }^{-2}$. Other expressions linking $A(\mathrm{FUV})$ and $X$ do exist (e.g., Burgarella et al. 2005) with subtle differences, and since this kind of calibration might also be subject to future revisions, we included in our figures both scales [observed $F_{\mathrm{TIR}} / F_{\mathrm{FUV}}$, calibrated $A(\mathrm{FUV})]$.

Given the long chain of operations in computing $A(\mathrm{FUV})$, we estimated in a simple way its uncertainty by computing the extremal values it can take when moving the UV and infrared fluxes 
by $1 \sigma$ from their observed values. FIR and UV data are moreover uncertain by $\sim 15 \%$ on average, (see, e.g., Morrissey et al. 2005 for an assessment of the quality of the GALEX photometry, and Rice 1993 for the photometry of IRAS extended galaxies).

The additional uncertainty in the calibration of the relation given in equation (3) itself is not taken into account. Points and error bars were not allowed to include negative attenuations. In the few cases where the $A(\mathrm{FUV})$ obtained in this way was unphysical (e.g., for numerical reason), the last consistent estimated error was used for points at larger radii [this only affects a few outer points where error bars are anyway large as can be seen in the $A(\mathrm{FUV})$ profiles given in the Appendix]. The FUV observed profiles were finally corrected for attenuation using $A$ (FUV), corrected for inclination, and converted to a star formation rate using the Kennicutt (1998b) calibration.

The inclination correction accounts for the fact that the thickness of a galaxy (and thus the amount of stars) along the line of sight has to be multiplied by cos (inc) ("inc" being the inclination of the galaxy) to obtain the same values for a face-on orientation (inc $=0$ ). The adopted inclination is given by the ratio of D25 and d25 from Table 1, assuming the objects are intrinsically circular. Note that this procedure assumes that we computed the correct $A$ (FUV) for the inclination of the galaxy. Panuzzo et al. (2003) showed that the relation between $A(\mathrm{FUV})$ and the $F_{\mathrm{FIR}} / F_{\mathrm{FUV}}$ ratio actually depends on inclination. Their fit for edge-on galaxies differs from the fit for moderately inclined galaxies by up to about 0.3 mag of extinction at $2000 \AA$. There are other reasons why we should be cautious about very inclined galaxies: (1) Our data points are separated by one element of resolution along the major axis. In the case of inclined galaxies, the ellipses along the minor axis will be closer to each other than the resolution. Outer regions might therefore be contaminated by inner regions. (2) In an edge-on disk, the amount of material along the line of sight is such that only a full radiative-transfer modeling can be used to reconstruct the UV to submillimeter spectral energy distribution of galaxies (e.g., Popescu et al. 2000). For this reason, our data are presented when possible with different symbols for various axis ratio ranges, and we discussed in the text when the inclination affected our results.

Corollary data were collected from the literature, concerning the gaseous content ( $\mathrm{H}$ I, $\mathrm{CO}$, rotation curve) and the oxygen abundance gradient of the galaxies. $\mathrm{CO}$ data were converted to molecular $\mathrm{H}_{2}$ using the conversion factor of Boselli et al. (2002) dependent on the metallicity when an abundance gradient was available. For the seven galaxies without metallicity gradient, we used the conversion factor corresponding to the $H$-band luminosity of each galaxy, using the calibration of Boselli et al. (2002).

The gas profiles taken from the literature were converted to the same inclination as mentioned before (indicated by the index "inc"). We also determined face-on values (index " 0 ") of the same quantities performing a similar correction than the one applied to the UV surface brightness (equivalent to a star formation rate surface density). Note that face-on values should be used to compare star formation rate and gas surface densities, while the extinction profiles obtained for the observed galaxy inclination should be compared to the column densities along the line of sight, i.e., the ones with the "inc" index. While we corrected the original gas data to have consistent inclination with our own UV and far-infrared computations, we must acknowledge that this compilation introduces some level of uncertainty since all observations were not performed with the same instruments, spatial resolution, etc. We, however, consider that this effect is included within the typical uncertainties discussed below.

Typical uncertainties are 0.15 dex for abundances, about $10 \%$ for $\mathrm{H}_{\mathrm{I}}$ data, and $50 \%$ for $\mathrm{H}_{2}$. The fact that we used a constant $\mathrm{CO}$ - to- $\mathrm{H}_{2}$ conversion factor when we had no abundance gradient for 7 galaxies must introduce an error in their molecular gas column densities. However, Figure 1 of Boissier et al. (2003) shows that usually the difference between a constant and a metallicitydependent conversion factor is lower than $0.5 \mathrm{dex}$. When computing the total gas density $\left(\mathrm{H} \mathrm{I}+\mathrm{H}_{2}\right)$, a significant number of points have only $\mathrm{H}$ I data, and the contribution of $\mathrm{H}_{2}$ was then assumed to be negligible. This of course introduces another uncertainty on the total gas density, however: (1) Most of those points correspond to outer regions of disks in which $\mathrm{CO}$ is detected and measured only for the inner portion of that same galaxy. It is reasonable to assume that the molecular fraction is small in these outer regions. (2) In 13 galaxies, we only have $\mathrm{H}$ I data (even in the center of the galaxy) but taking into account that only a minor fraction of the total gas is usually in the form of molecules (about $15 \%$, see Boselli et al. 2002) and that when we do have both profiles, $\mathrm{H}_{2}$ dominates only for a few central points, and in most cases by only a factor a few; we must conclude that the error we commit is relatively small in most cases. We will nevertheless keep in mind these drawbacks when discussing our results. When we found several sets of data, we usually adopted one (that seemed bettermore recent, better sensitivity) and checked the consistency between them. In a few cases, we adopted an average or a combination of several references. Note that we interpolated the profiles given in the literature at the resolution adopted in our work. The original resolutions are very variable but usually better than the IRAS one. The gaseous profiles obtained following this procedure are given in the figure set in the Appendix for each individual galaxy (at the same resolution as for the rest of our work). Table 1 gives the references for all of these data.

\section{ATTENUATION IN THE DISK OF LATE-TYPE GALAXIES}

\subsection{Attenuation Radial Profiles}

The attenuation $A$ (FUV) profiles are shown for each individual galaxy in the Appendix. Most of them present a global decrease of extinction with radius. At large radii, error bars are very large due to low levels of measured infrared emission, so that the data are always consistent with no attenuation in the outer disks. The central value of the estimated FUV attenuation is typically of a few magnitudes (ranging from virtually 0 to about 6 mag). The most central point of our profiles could be affected by the presence of a bulge with, e.g., UV upturn, or of an AGN. The fact that we see in the individual $A$ (FUV) profiles continuous trends with radius let us think that on average our extinction gradients are not much affected by such cases [in the figure set of the Appendix, only M31 and NGC 0253 do present a strong disturbance in the central part of the $A(\mathrm{FUV})$ profile].

A similar trend of decreasing extinction gradients was present in the six galaxies of the FOCA/IRAS study of Boissier et al. (2004) and in M101 (Popescu et al. 2005). Holwerda et al. (2005b) used counts of distant galaxies, with a "synthetic field method" (Holwerda et al. 2005a) to estimate extinction gradients. Their totally independent results are consistent with ours, although the uncertainties are larger in their method.

At this point, we would like to include some warning concerning the derived extinction $A$ (FUV). By adopting the Buat et al. (2005) calibration, we assume that the star formation histories they adopted are representative of real galaxies. However, it is probably not the case for a few galaxies (or at least, parts of galaxies) suffering heavy quenching of the star formation rate (that could be induced, for instance by ram pressure in clusters, e.g., Boselli et al. 2006). In such galaxies, the GALEX FUV - NUV 

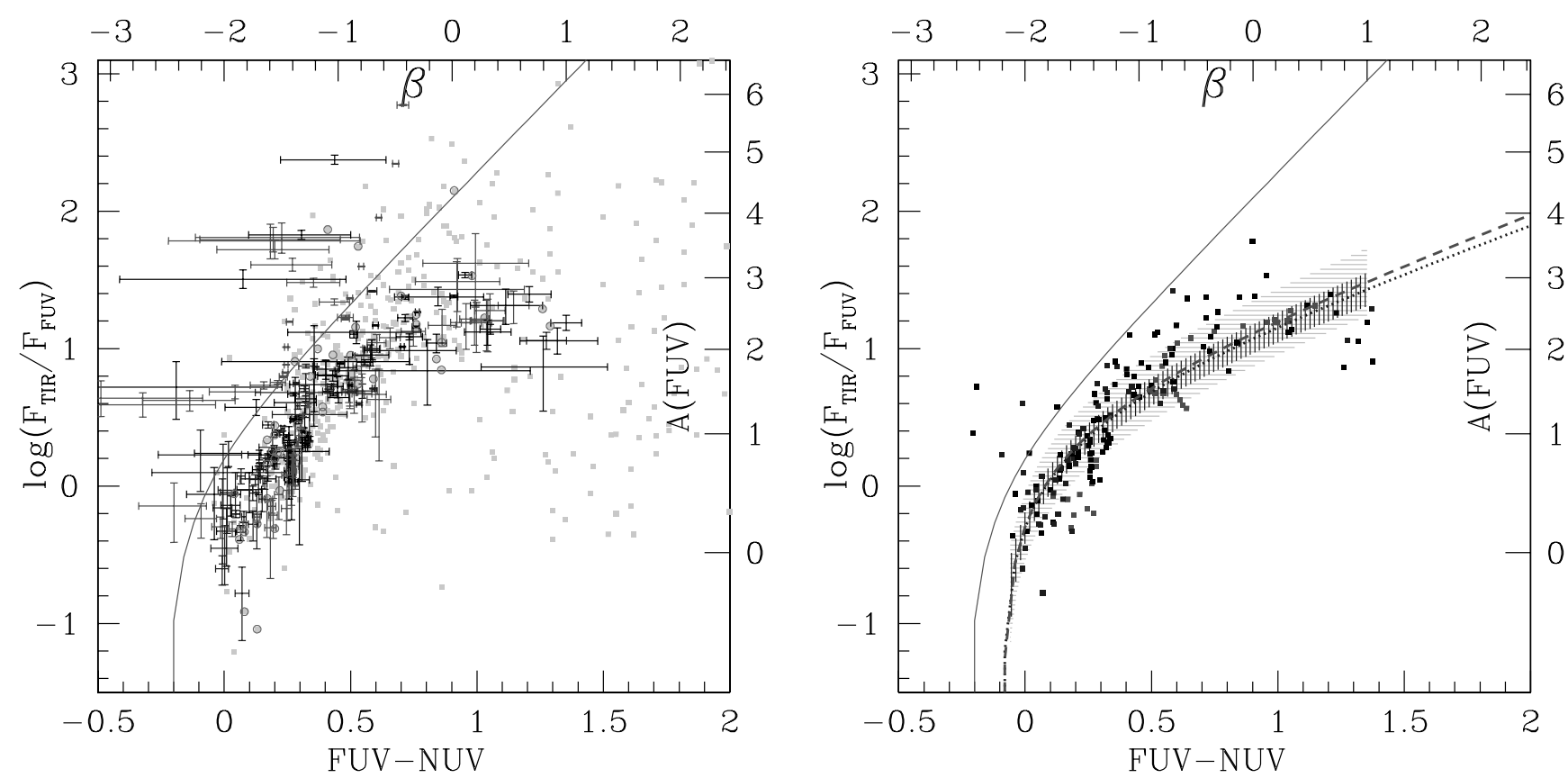

Fig. 1. - Total infrared to FUV ratio vs. FUV - NUV color. The FUV - NUV color is a measure of the slope in the UV $\beta$ given at the top (Kong et al. 2004). The right axis indicates the scale in $A$ (FUV) using eq. (3). In the left panel, points with error bars larger than 0.5 (dex on left axis and mag on bottom axis) were omitted for the sake of clarity (error bars indicate how much the results would change if our sky determination in the UV or far-infrared was moved by $\pm 1 \sigma$ ). The line is the usual relation for starbursts as given in Kong et al. (2004). Gray squares are the integrated values of the GALEX Atlas of Nearby Galaxies (Gil de Paz et al. 2007). Squares with a circle correspond to the galaxies in our study. In the right panel, we show as black squares the points used to compute our fits (excluding nearly edge-on galaxies). The usual starbursts relationship is compared to our fit and its $1 \sigma$ deviation for a simple fit (dashed curve and horizontally hatched area) and a fit taking into account the uncertainties (dotted line and vertically hatched area). [See the electronic edition of the Supplement for a color version of this figure.]

color would be intrinsically red (close to or above 1 ), with a different relationship between $F_{\mathrm{TIR}} / F_{\mathrm{FUV}}$ and $A(\mathrm{FUV})$ than the one we adopted. Such situations will be studied in L. Cortese et al. (2008, in preparation). Part of the scatter in the right part of Figure 1 could be due to such effects. As a result, applying extinction correction to individual galaxies (or profiles) should still be done with caution, especially for red FUV - NUV colors. In our approach, the number of galaxies is sufficiently large so that such effects should be minor on our global results.

\subsection{The Infrared Excess-UV Slope (IRX- $\beta$ ) Relationship}

Heckman et al. (1995) and Meurer et al. (1995, 1999) demonstrated with IUE data the existence of a relation between the slope of the UV spectrum $(\beta)$ and the TIR/UV ratio (so-called IRX- $\beta$ relationship) for starburst galaxies. It has been common thereafter (especially for high-redshift objects) to estimate the extinction from the UV slope using the IRX- $\beta$ relationship (e.g., Schiminovich et al. 2005). In the recent years, however, several studies have shown that this relation does not hold for all galaxies. For example, Bell (2002) showed that it fails in nearby galaxies, and Buat et al. (2005) came to the same conclusion for samples of UV and far-infrared-selected galaxies. Kong et al. (2004) proposed models with a large variety of star formation histories, demonstrating that they could span a large portion of the IRX- $\beta$ plane. In this framework, the starburst relation would hold for galaxies with extremely large current-to-past-average star formation rates (birthrate $b$ parameter), while galaxies with less activity would lie systematically below the relation. However, it seems that a relation can be found in nearby galaxies (see, e.g., Gil de Paz et al. 2007; Cortese et al. 2006; Seibert et al. 2005 ), given a simple offset with respect to the original relation proposed for starbursts. This means that the range of star formation histories among real galaxies is probably not as wide as among models of Kong et al. (2004) and that the $b$ parameter might not be the driver of the IRX- $\beta$ relationship. Actually, Burgarella et al. (2005) showed that the slope of the extinction and the presence or not of a bump has a larger impact on the position in the IRX- $\beta$ plane than the star formation history (and the $b$ parameter). As discussed in Gil de Paz et al. (2007), Cortese et al. (2006), and Seibert et al. (2005) the difference in the IRX- $\beta$ relation for starbursts and normal galaxies might be due to geometrical effects, aperture effects present in the IUE data. The relative calibrations in the computation of $\beta$ with IUE's data and GALEX FUV NUV color index could also play a role. More work remains to be done.

In this context, our nearby galaxies have the advantage of being close enough so that we can spatially resolve the IRX- $\beta$ relationship. While this has been done by looking at individual regions in a few galaxies already, for instance M51 (Calzetti et al. 2005 ), we present for the first time the IRX- $\beta$ relationship for the profiles of all the large late-type galaxies of the GALEX Nearby Galaxies Atlas in Figure 1. It is particularly interesting to note that, while outliers are present (with correspondingly larger error bars), most of the points describe a very tight relation, but shifted from the classical relation derived for/from starbursts, similar to the recent works of Gil de Paz et al. (2007), Cortese et al. (2006), and Seibert et al. (2005; see discussion above for the possible reasons for this shift). In order to quantify the relationship, we performed a nonlinear least-squares fit of the form $y=\log \left(10^{a+b x}-c\right)$ (with $x=\mathrm{FUV}-\mathrm{NUV}$, the color between the two GALEX bands, and $y=\log \left(F_{\mathrm{TIR}} / F_{\mathrm{FUV}}\right)$, after rejecting quite edge-on galaxies (NGC 0055, NGC 0253, NGC 0660, NGC 0891, NGC 4216, NGC 4621, NGC 4656a). We obtained for $a, b$, and $c$, respectively, $0.570,0.671$, and 3.220 using $y$-uncertainties as weight, and $0.561,0.713$, and 3.136 using no weights. These fits and their associated $1 \sigma$ dispersion are shown in the right part of Figure 1.

The fact that our IRX- $\beta$ relationship is quite tight is also interesting as there is much more scatter when individual regions are 

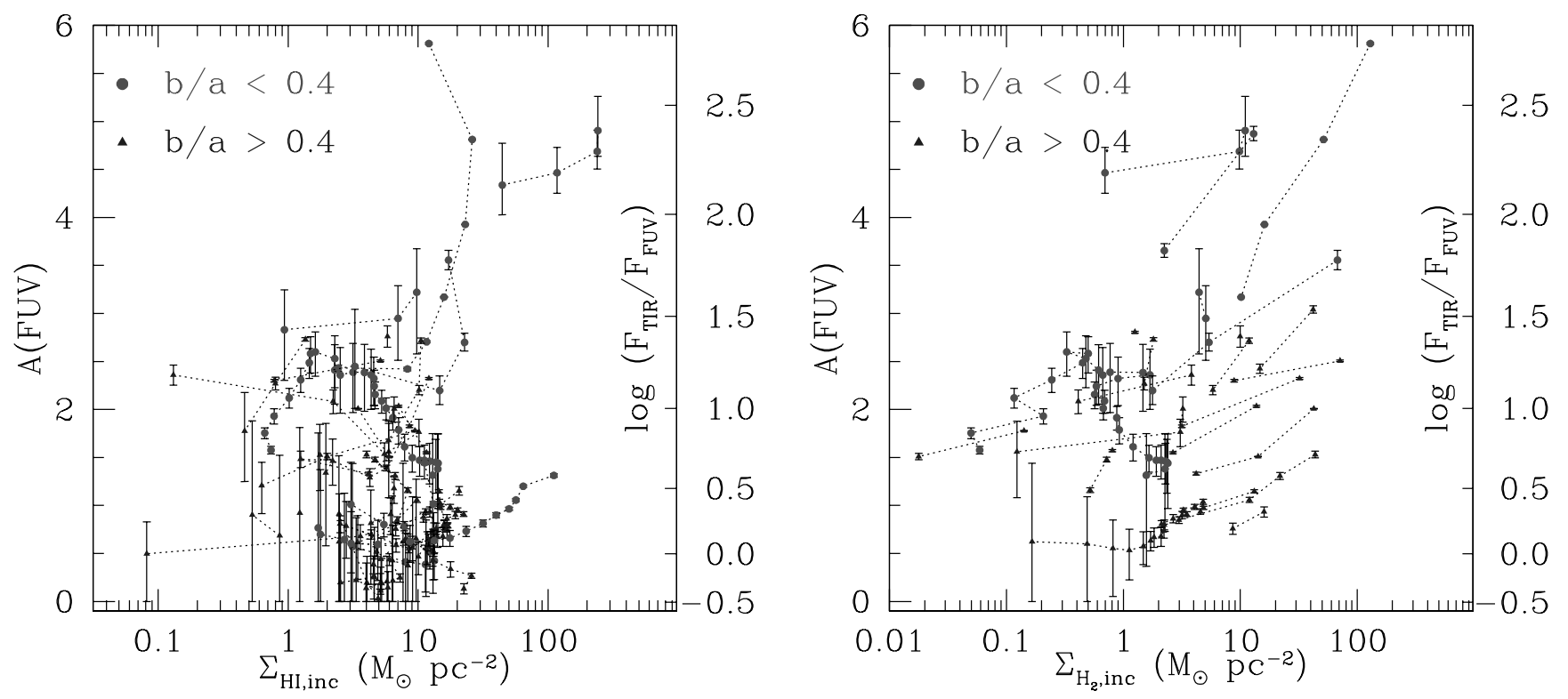

FIG. 2.-Attenuation at FUV wavelength $\left(F_{\mathrm{TIR}} / F_{\mathrm{FUV}}\right.$ ratio scale on the right axis) as a function of the observed column density of $\mathrm{H}$ I $(l e f t)$, $\mathrm{H}_{2}($ right $)$. Points belonging to a same galaxy are connected by a dotted line. Different symbols indicate different values of the axis ratio $(b / a)$ as indicated in the corner. Note that since $A(\mathrm{FUV})$ is not corrected for inclination, the gas column densities used correspond to the same inclination (hence the "inc" subscript). Error bars indicate how much

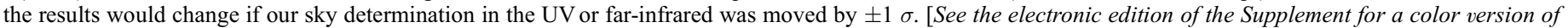
this figure.]

looked at, see for instance Calzetti et al. (2005) in M51a, or Thilker et al. (2007) in NGC 7331, both studies based on the GALEX/SINGS joint analysis of GALEX and Spitzer data from the SINGS project (Kennicutt et al. 2003b). By azimuthally averaging profiles, we remove the effects due to small-scale starformation histories (affecting the UV slope) and we are likely to reduce the effect of radiative transfer peculiarities on small scales: that is, the dust in some small regions may well be heated by the UV emission from neighboring regions. Using the low resolution of IRAS (coupled with azimuthally averaging) may turn out to be an advantage in this respect.

Figure 1 also presents the integrated values for the galaxies of the Atlas (Gil de Paz et al. 2007) as gray squares. The squares overplotted with a circle correspond to the integrated value given in the Atlas for the galaxies of the present study. The relation of our study seems tighter than the one for the integrated galaxies of the Atlas. This can be explained by the combination of several effects: (1) For all our galaxies, we followed exactly the same procedure from scratch, i.e., we do not use published fluxes but rather rederive them ab initio, in contrast the UV Atlas for which fluxes were compiled from a wide variety of sources. (2) In integrated galaxies, centers with activity might affect the whole flux if they are strong enough, while they probably influence only the central point in a profile like the one we compile. About half of our galaxies have a sign of central activity (AGN, LINER, Seyfert indicated in NED), but precisely because it is confined to the central point, it should not affect our profiles much.

\subsection{The Absence of a Trend between Attenuation and Gas Column Density}

It is relatively common to consider the attenuation to gas density ratio as being constant. For instance, Komugi et al. (2005) adopt $N_{\mathrm{H}}=2 \times 10^{21} A_{V}$. This is essentially inspired by studies in the Milky Way such as Bohlin et al. (1978). Under this assumption, one would expect a strong correlation between gas density and attenuation, which is not supported by our results (see Figs. 2 and 3). Thus, attenuation (and therefore the existence of dust) is probably affected by various processes not directly related to the gas density. In Figure 2, we show the amount of extinction as a function of the gas in the form of neutral hydrogen ( $\mathrm{H}$, left $)$ and in the form of molecular hydrogen $\left(\mathrm{H}_{2}\right.$, right $)$. Within individual galaxies ( points connected by one dotted curve), a trend of increasing attenuation with gas densities is generally visible (specially with $\mathrm{H}_{2}$ ). However, the differences from one galaxy to another are large, so that globally no relation is clearly emerging.

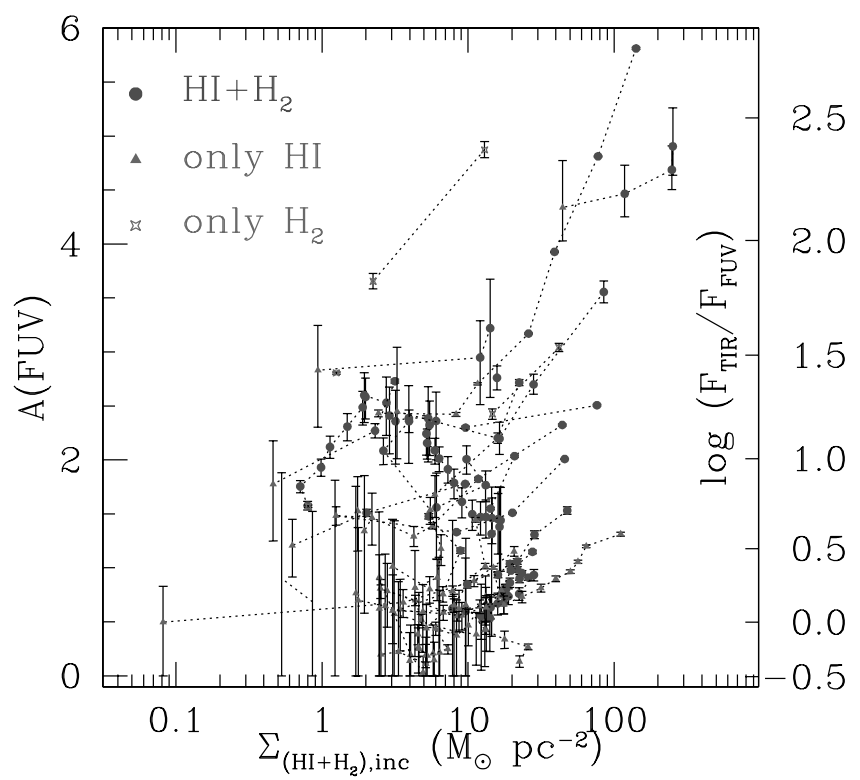

FIG. 3.- Attenuation at FUV wavelength $\left(F_{\mathrm{TIR}} / F_{\mathrm{FUV}}\right.$ ratio scale on the right axis) as a function of the observed total gas density $\left(\mathrm{H} \mathrm{I}_{\mathrm{I}}+\mathrm{H}_{2}\right)$. Since $A(\mathrm{FUV})$ is not corrected for inclination, the gas column densities used correspond to the same inclination (hence the "inc" subscript). Error bars indicate how much the results would change if our sky determination in the UV or far-infrared was moved by $\pm 1 \sigma$. [See the electronic edition of the Supplement for a color version of this figure.] 


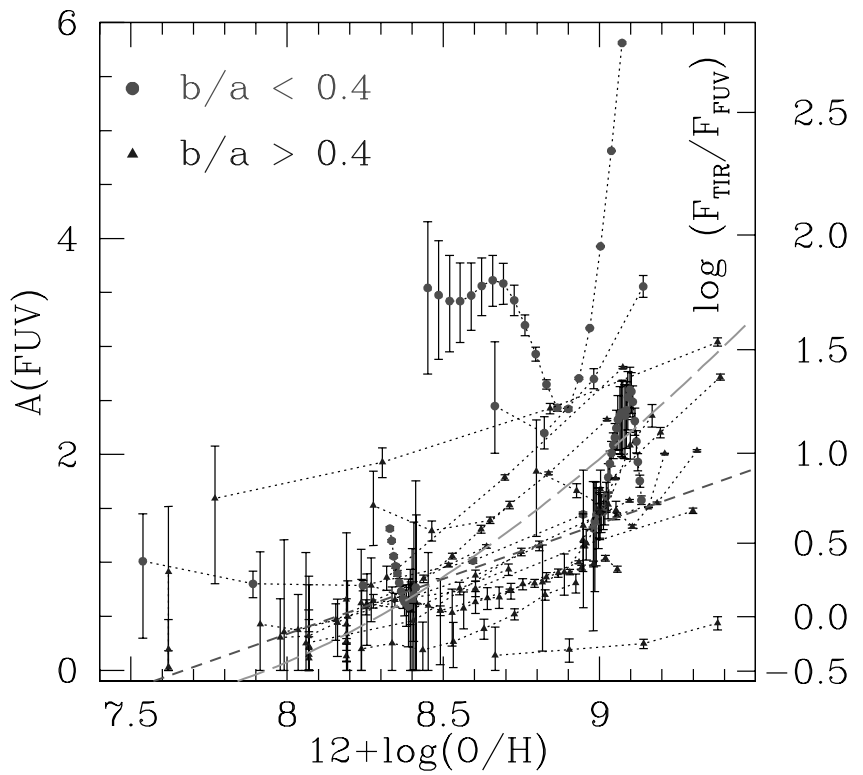

FIG. 4.- Attenuation at FUV wavelength $\left(F_{\mathrm{TIR}} / F_{\mathrm{FUV}}\right.$ ratio scale on the right axis) as a function of the metallicity (as given by the abundance gradient of each galaxy). The short-dashed line is a fit $\{A(\mathrm{FUV})=1.02[12+\log (\mathrm{O} / \mathrm{H})]-7.84\}$ to the points belonging to galaxies with $b / a>0.4$ (see $\delta 3.4$ ). The long-dashed curve corresponds to the fit of $F_{\mathrm{TIR}} / F_{\mathrm{FUV}}$ vs. $\mathrm{O} / \mathrm{H}$ in Cortese et al. (2006). Error bars indicate how much the results would change if our sky determination in the UV or far-infrared was moved by $\pm 1 \sigma$. [See the electronic edition of the Supplement for a color version of this figure.]

The fact that the amount of attenuation does not seem to correlate with the $\mathrm{H}$ i column density was also found by Holwerda et al. (2005b). A small trend can be seen between the molecular gas and the extinction. While this seems reasonable (inner regions are denser, have larger extinction and higher molecular fraction) and is observed in most galaxies individually, the variations from one galaxy to another are still extremely large. In Figure 3, we compare the ultraviolet attenuation $A$ (FUV) to the total gas density. In this case, we are in an intermediate situation. At low gas column densities, which correspond usually to the outer part of galaxies (where errors are larger), no trend is observed. In the inner parts of galaxies, the situation is the same as for $\mathrm{H}_{2}$ since the molecular gas often dominates the total gas density or at least appears to compensate for the central plateau or the observed decrease of $\mathrm{H}_{\mathrm{I}}$ in the central regions of galaxies.

In conclusion, our data do not support any relation of the type $A / N_{\mathrm{H}}=$ constant (commonly used in the literature). This is actually not surprising as $A$ (FUV) is not a measure of the dust mass, but of the amount of dust heated by nearby young massive stars. As such, it will be largely affected by geometrical effects, and the physical properties of the dust grains (type, size distribution, ...), which would probably erase any underlying relation between the dust and gas masses. Because the dust mass is dominated by cold dust, $A(\mathrm{FUV})$ is just not a good measure of it. Estimating the dust mass, and thus the dust-to-gas mass ratio is out of the scope of this paper since IRAS wavelengths do not allow us to probe the cold dust.

\subsection{The Attenuation-Metallicity Relationship}

While the relation observed with the gas is ill-defined, the attenuation seems to depend in a clearer way on the metallicity (Fig. 4). Indeed, for a given abundance of oxygen, the scatter in observed extinction is smaller than at any given gas surface density. This is especially true if we exclude the galaxies with the largest inclinations in which the method used to derive the attenuation might suffer from various problems as commented in $\S 2$. We performed a least-squares minimization fit to the points within galaxies with axis ratio larger than 0.4 and found

$$
A(\mathrm{FUV})=1.02 \times[12+\log (\mathrm{O} / \mathrm{H})]-7.84
$$

(the dispersion around this fit is $\sigma_{A(\mathrm{FUV})}=0.5 \mathrm{mag}$, and the correlation coefficient is 0.65 ).

This is qualitatively consistent with the earlier work of Boissier et al. (2004). Albeit a bit shallower, this relation is characterized by similar attenuations in the high-metallicity range [at $12+$ $\log (\mathrm{O} / \mathrm{H}) \sim 9.3]$. Due to the limited number of galaxies, there were no points at the lower metallicities $(7.5-8.5)$ in Boissier et al. (2004). A source of potential difference between this earlier study and the present one is that it was performed at the FOCA wavelength rather than GALEX. In Boissier et al. (2004) the extinction determined in the integrated star-forming galaxies of Buat et al. (2002) with metallicities of Gavazzi et al. (2004) were also shown to exhibit a similar trend. Our results are also in good agreement with the recent study of Cortese et al. (2006) (although their trend is slightly stronger than ours), for a sample of integrated cluster galaxies observed with GALEX [their fit of the TIRto-FUV ratio is converted to our $A$ (FUV) scale in Fig. 4].

Note that the attenuation-metallicity relationship we found concerns galaxies forming stars in a relatively quiescent (disk)-mode. Heckman et al. (1998) have shown that the UV slope is correlated with the metallicity in starbursts, also corresponding to a metallicityextinction relationship among starburst, shifted however with respect to the spirals to higher extinctions (and presented in Boissier et al. 2004).

\section{STAR FORMATION LAW}

\subsection{The Theoretical Star Formation Laws}

Star formation on galactic scales is obviously a crucial phenomenon in the evolution of galaxies. As a result, it is also a fundamental element of any galaxy evolutionary model, e.g., $N$-body/ semianalytical models like GALICS (Hatton et al. 2003), SPH/ chemical evolution models (Lia et al. 2002), chemical/spectrophotometric evolution models (Boissier \& Prantzos 1999). Despite its importance, only rough theories exist and a few empirical relationships have been looked for (e.g., Madore 1977; Buat et al. 1989; Kennicutt 1998a; Wong \& Blitz 2002). An excellent review of the empirical situation is given in Kennicutt (1998b), while Elmegreen (2002) consider the possible physical origin of the empirical laws. The reader is refereed to these works for an extensive discussion on the topic. Here, we only come back rather quickly on a few of the relations proposed for the "star formation law." These "laws" should allow us to predict the star formation rate from other physical quantities, as shown below. Especially, we will look for relations between the star formation rate surface density $\left(\Sigma_{\mathrm{SFR}}\right)$ and the gas surface density in its various phases (neutral, molecular) as well as in total gas (neutral + molecular), under the form of a traditional "Schmidt law" (see Schmidt 1959 for the original work):

$$
\Sigma_{\mathrm{SFR}}=\alpha \Sigma_{\mathrm{GAS}}^{n}
$$

It is reasonable to expect a more direct relationship with the molecular gas rather than the neutral gas since this phase is more closely in the sequence related to the ensuing star formation event. 
For instance, in their disk model consisting of self-gravitating clouds interacting to produce a turbulent viscosity, Vollmer \& Beckert (2002) found that the star formation rate and molecular gas surface densities should follow the same dependence with galactic radius. On the observational side, while a relation between star formation rate and molecular mass was not initially confirmed (Kennicutt 1989; Boselli et al. 1995), Boselli et al. (2002) showed that using a luminosity or metallicity-dependent CO-to- $\mathrm{H}_{2}$ conversion factor provides a better relation than using $\mathrm{H}$ I alone.

While this suggests a more direct connection between the star formation rate and the molecular gas, the cause for star formation could still be related to the total gas. Indeed, a basic model of self-gravitating disk would suggest a Schmidt law with the total gas density as the main control parameter (Kennicutt 1998a).

The other model we will consider here is a Schmidt law modulated by a dynamical factor:

$$
\Sigma_{\mathrm{SFR}}=\alpha \Sigma_{\mathrm{GAS}}^{n} \frac{V(R)}{R},
$$

where $V(R)$ is the rotational velocity at radius $R$ (where the surface densities are determined). The ratio $V(R) / R$ can be considered either as representing the frequency of the passage of density waves enhancing star formation (Ohnishi 1975; Wyse \& Silk 1989) or a dynamical timescale (Kennicutt 1998b). In their model of the chemical evolution of the Milky Way, Prantzos \& Aubert (1995) found that an another factor in addition to a simple Schmidt law was necessary and opted for a relation of the type given by equation (6) with $V(R)=$ constant, and $n=1$. Subsequent models of the Milky Way and spirals used a similar SFR law (Boissier \& Prantzos 1999; Boissier \& Prantzos 2000) but with $n=1.5$. This was found to give agreement with the $\mathrm{H} \alpha$ profiles of 16 nearby spirals studied by Boissier et al. (2003), although other laws could not be excluded. Boissier et al. (2003) had also tested a dependence on the stellar surface density in addition to the gas, as proposed by Dopita \& Ryder (1994). Despite having an additional free parameter, this formulation did not offer a better fit to their data. To explore this further we would need near-infrared surface brightnesses for our galaxies. Unfortunately, 2MASS data are not sufficiently deep in outer parts of our galaxies, and we decided to omit consideration of this law in our present study.

Before testing the above proposed laws, we should note that the field of galactic star formation is not lacking new ideas. For instance, Elmegreen (2002) proposed that a combination of processes trigger star formation, conforming to the observed relations and thresholds (densities below which no star formation would occur). Seigar (2005) presented an interesting correlation between the specific star formation rate and the shear rate, stressing the possible role of shear in star formation; however, the shear versus average star formation rate (his Fig. 4) has large dispersion. Our rotation curves do not have the necessary resolution or homogeneity to explore trends involving the shear rate. It has also been recently suggested (Barnes 2004) that star formation may depend not only on the local gas density but may also be induced by shocks, especially in the case of interacting galaxies (we do not have such violently interacting systems in our sample). Testing this kind of star formation law requires detailed dynamical modeling on a caseby-case basis. Barnes (2004) showed that this idea is promising by reproducing observations of the Mice (NGC 4676). However, it would be hard to implement such a law in, e.g., semianalytic model since it needs a detailed dynamical modeling of each interacting systems (including their precise geometry and details of the encounter).
In any case, a best possible law for star formation should still properly reproduce the relation observed between gas, dynamics, and star formation indicators that we compiled for our GALEX galaxies, whatever the physical causes are. Thus, this purely empirical work provides additional constraints for future theoretical work and galaxy evolution models.

\subsection{Our Constraints on the Star Formation Laws}

In each panel of Figure 5 we show a variant of the simple Schmidt law (SFR vs. neutral gas, molecular gas, total gas), testing equation (5) and a combination of the SFR + dynamical factor versus total gas testing the relation of equation (6). In this figure, the internal contributions of individual galaxies are connected by dotted lines. Individual SFR and gas profiles, as well as SFR versus gas are given for each galaxy individually in the Appendix. Correlation coefficients are indicated in each panel, as well as the two (dashed) regression lines whose coefficients are given in Table 2. For each relation, the first regression line is obtained by performing a least-squares fit of the star formation rate surface densities with the gas surface densities being given (minimizing the errors on the $Y$-axis), and the other one by performing a least-squares fit of the gas surface densities with the star formation rate surface densities being given (minimizing the errors on the $X$-axis).

It is obvious from this figure that the form of the observed star formation laws differs from one spiral galaxy to another. This was already known and discussed earlier (e.g., Boissier et al. 2003; Kennicutt 1998a). It is hoped that averaging over many galaxies is equivalent to averaging the radial star formation history of any given galaxy over time. The trend with $\mathrm{H}_{2}$ seems relatively good on the high-density side (larger than $\sim 1 M_{\odot} \mathrm{pc}^{-2}$ ), but the number of points with molecular gas alone is very small, and the results are inconclusive, especially given the additional uncertainty on the conversion factor. As in the case of extinction versus $\mathrm{H}_{2}$, however, the scatter seems too large to be caused by this factor alone. We note that Komugi et al. (2005) show that $\mathrm{H}_{2}$ is very well correlated with the star formation rate but they are interested in high-density regions and galactic nuclei, working at high resolution. In our case, to the contrary, the central parts of the galaxies have little influence in our plots since we are working at low resolution and looking at the largest possible radii. When the total gas is used, as in the study of the attenuation, we acknowledge that the absence of molecular gas data can affect the precise value of the slope and the intercept; however, the scatter seems too large to be entirely due to the uncertainties in the $\mathrm{H}_{2}$ content alone. Boissier et al. (2003) showed that the extrapolation (or not) of CO data, and the use of a metallicity-dependent or a constant conversion factor did change their results significantly, but mostly influenced the star formation threshold (without much affecting the rate exponent $n$ ).

Among all of the Schmidt law variants that we tried, the simple Schmidt law (with a dependence on the total gas density) gives the best results, although the correlation coefficient for the molecular gas and the "dynamical" law (0.67 and 0.62, respectively) are close to the one for the total gas (0.68). Komugi et al. (2005) have shown that the simple Schmidt law extends to higher gas surface densities when smaller and central regions (assumed to be dominated by $\mathrm{H}_{2}$ ) are considered. If added to our diagrams, their data would go from about 10 to $1000 M_{\odot} \mathrm{pc}^{-2}$ for the gas, corresponding to a range in star formation rate from about 1 to $1000 M_{\odot} \mathrm{pc}^{-2} \mathrm{Gyr}^{-1}$. This is compatible with an extrapolation of our adopted Schmidt law (using the total gas) to larger surface densities, with a similar slope. We notice, however, a shift to lower star formation rates for the same gas density. In their 

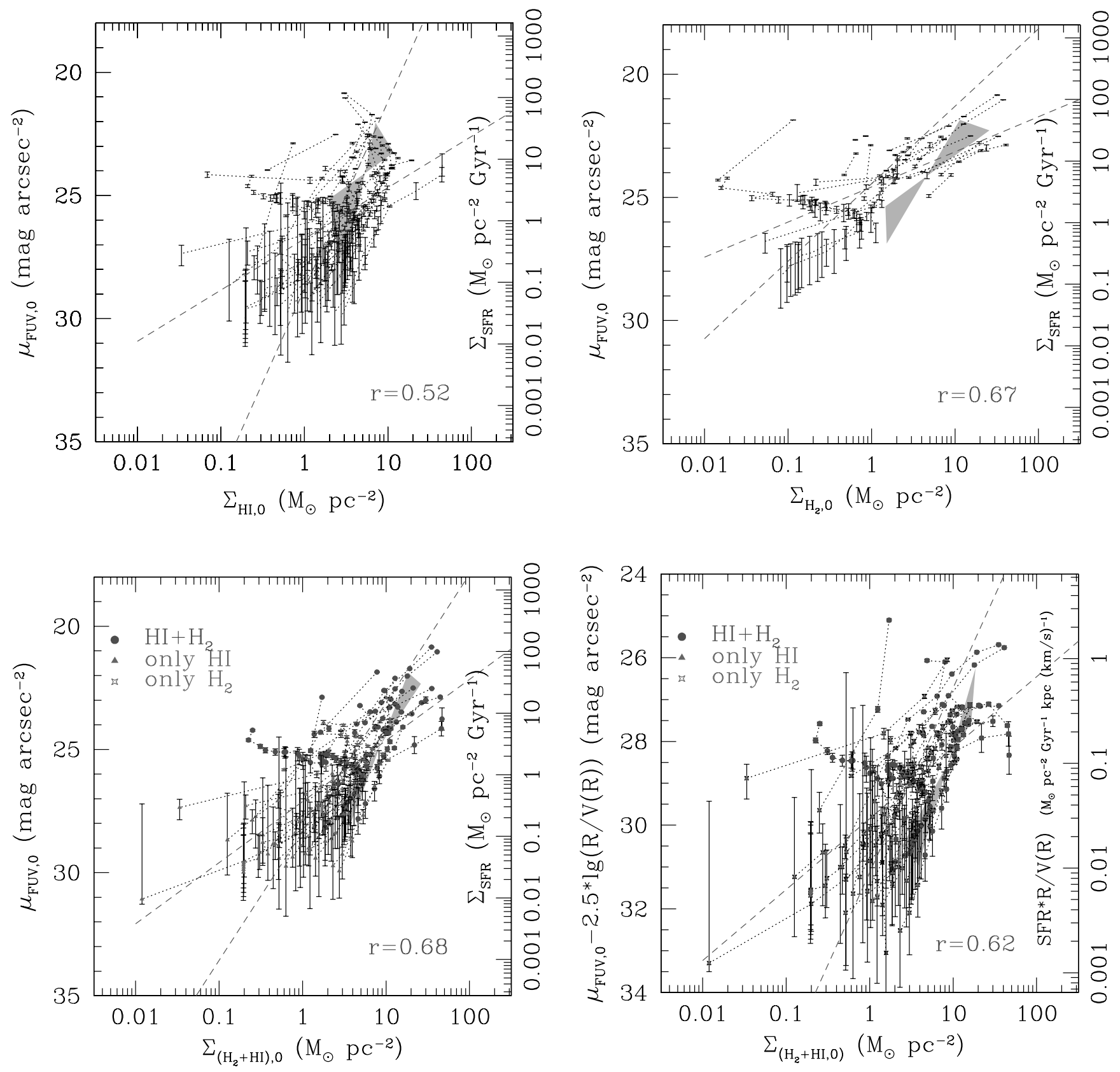

FIG. 5.- Test of various "Schmidt laws" for our whole sample. The star formation rate (right axis) derived from FUV profiles corrected for attenuation and inclination is compared to $\mathrm{H}$ I (top left), $\mathrm{H}_{2}$ (top right), total $\mathrm{H}$ surface densities (bottom left). In the bottom right panel, we show the $\mathrm{SFR} \times R / V(R)$ vs. hydrogen total density to test eq. (6). In each box, the hourglass shaped shaded area indicates equivalent results from Boissier et al. (2003) based on H $\alpha$ profiles (the extent corresponds roughly to the extent of the observations and the diagonals to the regression lines of this study). The correlation coefficient and regression lines (dashed lines, obtained fitting $y$ vs. $x$ and $x$ vs $y$; see $\S 4.2$ ) are shown (their parameters are given in Table 2). Error bars indicate how much the results would change if our sky determination in the UV or far-infrared was moved by $\pm 1 \sigma$. [See the electronic edition of the Supplement for a color version of this figure.]

TABLE 2

Parameters $a_{i}$ and $b_{i}(i=1,2)$ of the Two Regression Lines $\left[\log (Y)=a_{i} \log (X)+b_{i}\right]$

\begin{tabular}{|c|c|c|c|c|c|c|c|}
\hline$X$ & $Y$ & $a_{1}$ & $b_{1}$ & $a_{2}$ & $b_{2}$ & $r$ & $N$ \\
\hline$\Sigma_{\mathrm{H}_{\mathrm{I}}} \ldots \ldots \ldots \ldots \ldots \ldots \ldots \ldots \ldots \ldots$ & $\Sigma_{\text {SFR }}$ & 0.83 & -0.30 & 3.04 & -1.11 & 0.52 & 232 \\
\hline$\Sigma_{\mathrm{H}_{2}} \ldots \ldots \ldots \ldots$ & $\Sigma_{\text {SFR }}$ & 0.57 & 0.58 & 1.26 & 0.63 & 0.67 & 108 \\
\hline$\Sigma_{\mathrm{H}_{\mathrm{I}}+\mathrm{H}_{2}} \ldots \ldots$ & $\Sigma_{\text {SFR }}$ & 0.99 & -0.44 & 2.09 & -0.92 & 0.68 & 236 \\
\hline$\Sigma_{\mathrm{H}_{\mathrm{I}}+\mathrm{H}_{2}} \ldots \ldots \ldots \ldots \ldots \ldots$ & $\Sigma_{\mathrm{SFR}} R / V(R)$ & 0.68 & -1.53 & 1.76 & -2.04 & 0.62 & 228 \\
\hline
\end{tabular}

Notes.-Parameters $a_{i}$ and $b_{i}(i=1,2)$ of the two regression lines $\left[\log (Y)=a_{i} \log (X)+b_{i}\right]$ shown as dashed lines in each panel of Fig. 5 (least-squares fit minimizing the errors on $X$ and $Y$, respectively). We also give for each set of variables $(X, Y)$ the correlation coefficient $r$ and the number of points used for the fit $(N)$. 


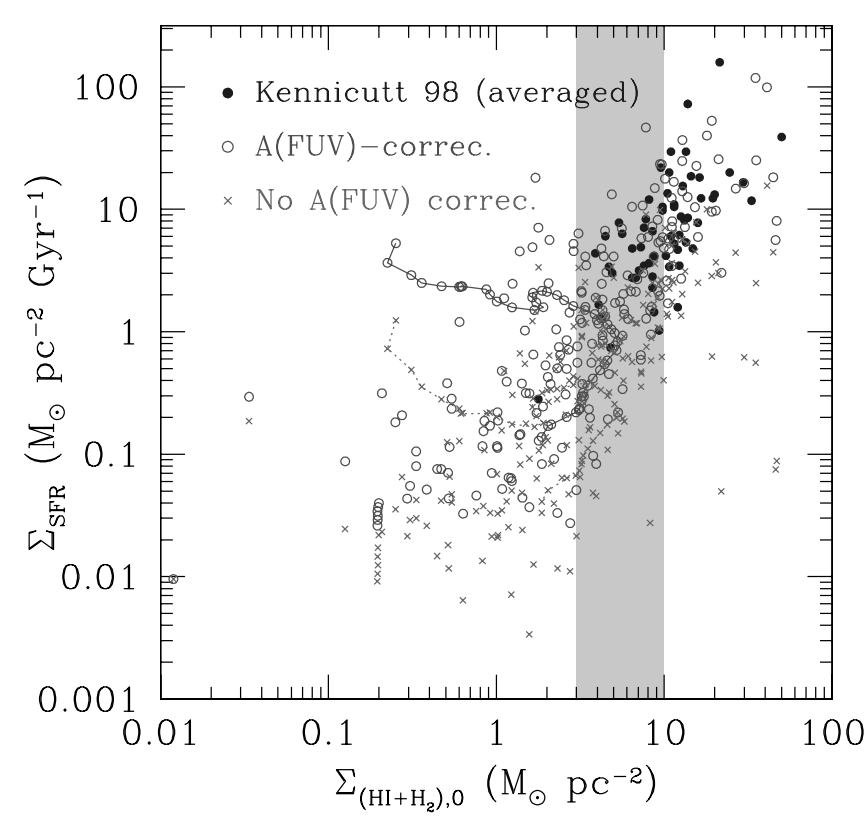

FIG. 6.- Simple Schmidt law (Star formation rate surface density vs. total hydrogen surface density) found in our work along radial profiles of GALEX galaxies (open circles, including the extinction correction; crosses, not including extinction) is compared to the one of Kennicutt (1998a) (filled circles, obtained by computing average surface densities within the optical disk). The shaded area indicates gas densities values found at the threshold by Kennicutt (1989). The studies overlap with each other in the high-density half, but we obtain many more points at low surface densities. The points corresponding to M31 (following an untypical path in the inner galaxy) are connected: high SFR and low gas amounts (top left) correspond to the inner galaxy. When increasing the radius, the SFR decreases while the gas increases (contrary to classical SFR "laws"). At about 50', the trend changes and both the SFR and the gas surface densities go down with radius, following the SFR "law" described by other galaxies. [See the electronic edition of the Supplement for a color version of this figure.]

study, the SFR is derived from $\mathrm{H} \alpha$, which might explain this offset (see below for a discussion on this point).

In all panels of Figure 5, we also show for comparison the results of Boissier et al. (2003) in the form of an hourglass-shaped shaded area (the extent of the hourglass being the extent of the observations, and the diagonals the regression lines found in this work). This study used $\mathrm{H} \alpha$ profiles for 16 spiral galaxies. There are some systematic differences: on average they found lower star formation rates for the same gas density than in our present study, except with H I. The slope of a simple Schmidt law was also slightly steeper than what we find. Overall, taking into account the large dispersion, the differences are not dramatic and may be caused by (1) inadequate/uncertain corrections: for instance, in Boissier et al. (2003) $\mathrm{H} \alpha$ was corrected for extinction and [N II] contamination by standard factors depending only on the galaxies type (a common procedure), but not considering possible radial variations, (2) calibration issues: the conversion of $\mathrm{H} \alpha$ and UV light to star formation rates depends on the initial mass function adopted for converting the observed fluxes (Kennicutt 1998b). For example, if the assumed IMF overestimates the number of ionizing stars, the star formation rate derived from $\mathrm{H} \alpha$ will be underestimated. Another difference that cannot be explained by these effects is the fact that the gas extent was smaller, i.e., with our UV profile, we detect low levels of star formation, corresponding to lower levels of gas surface densities.

In Figure 6, we compare the simple Schmidt law (obtained from our UV radial profiles vs. total gas) to the galaxies of Kennicutt (1998a) obtained from $\mathrm{H} \alpha$ imaging of spiral galaxies, averaged within their optical disk. Considering the scatter, the agreement between both studies is quite good. Here again, however, we have many more points at low gas densities. We also include in this diagram the star formation rate values derived from the UV assuming zero attenuation (crosses). These are lower limits on the star formation rate, showing that a significant amount of star formation is detected at low gas densities, and this result does not depend on the attenuation correction. This comes from several facts: (1) Kennicutt's estimates are averages within $R_{25}$, where densities are on average higher; (2) in the UV, we do find emission at low levels of star formation (in stark contrast to $\mathrm{H} \alpha$ observations), corresponding to gas surface densities below the putative threshold for star formation.

In this figure, we also connected the points corresponding to M31. The inner $50^{\prime \prime}$ of this galaxy are orthogonal to the usual Schmidt relation (and the one found in the rest of the sample). We leave it to later studies to interpret this untypical behavior of one of our nearest neighbors.

Our plots might appear more highly dispersed than other studies (e.g., Komugi et al. 2005; Kennicutt 1998a), but this is mainly because we do not work on a large range of gas densities (i.e., the scatter only seems smaller in Kennicutt (1998a) because of the larger dynamic range introduced into the plots when circumnuclear starbursts are included), and we are working primarily at low densities where stochastic effects and larger uncertainties may have a larger impact. Another possible cause of the dispersion is that we are using UV data, and as Buat et al. (1989) noted, the timescales we are probing are significantly longer than with $\mathrm{H} \alpha$, probably making the underlying "causal" relations harder to catch (Madore 1977).

\subsection{Concerning the Notion of a Threshold}

Star formation rate profiles derived from $\mathrm{H} \alpha$ data are known to frequently present an abrupt break, while atomic gas is generally much more spread than the star forming and the stellar disk (e.g., Martin \& Kennicutt 2001). This observation is traditionally interpreted as the result of local instabilities developing in differentially rotating disks within a threshold radius where the gas density is larger than a critical density (Toomre 1964). More complex physics has also been suggested (e.g., the onset of thermal instability of Schaye 2004).

The above-described observation of a Schmidt-like behavior at very low gas densities as derived from UV radial profiles suggests that low levels of star formation are in fact common beyond the usual threshold radius, where $\mathrm{H} \alpha$ emission ceases or is not (as easily) observed. We can verify that this is actually the case by taking the 9 galaxies for which we have UV and infrared data, $\mathrm{H} \alpha$ profiles from Martin \& Kennicutt (2001), and for which the threshold radius given in Martin \& Kennicutt (2001) is larger than $90^{\prime \prime}$. This last condition ensures that we would resolve the threshold at our low resolution.

The star formation rate profiles derived from the UV and the $\mathrm{H} \alpha$ emissions are presented in Figure 7. Within the threshold radius $R_{\text {threshold }}$, they are in rough agreement, while a systematic difference occurs beyond it: eight out of the nine galaxies show no truncation (or any change of slope) in the UV profiles at the position of the $\mathrm{H} \alpha$ threshold. The last one is M81, for which our UV profiles end at the position of the threshold. Note that we are limited for this galaxy by its large angular size: the south outer part of the galaxy is getting close to the limit of the GALEX field of view. The infrared background is also relatively bad so that we cannot extend the profiles very far out in the disk. However, an inspection of the FUV image (at full resolution) clearly reveals two spiral arms extending in the area between $R / R_{\text {threshold }}=1$ and 1.5. 


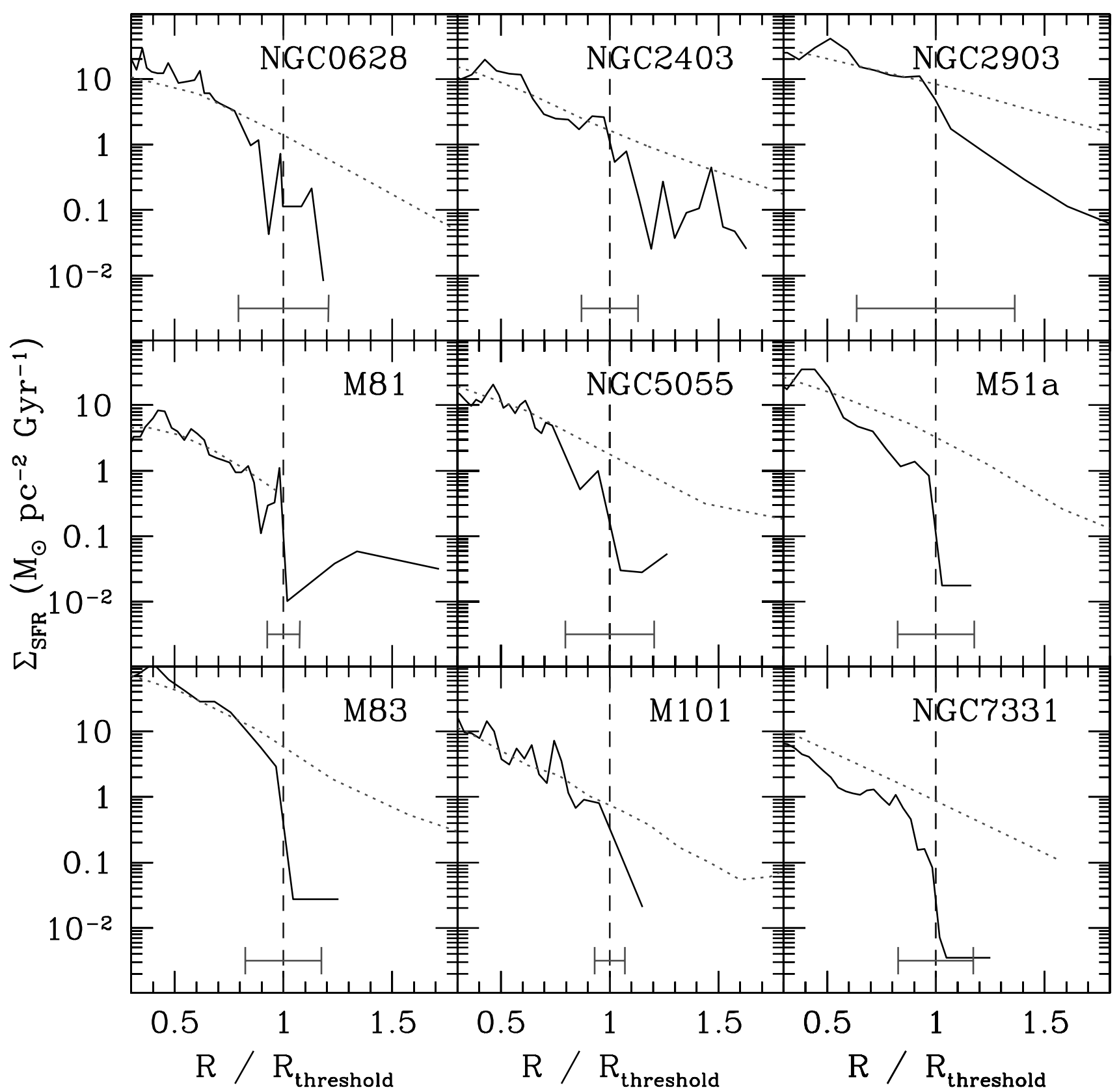

Fig. 7.- Profiles of star formation rate surface density determined from the UV and corrected for extinction with the far-infrared (dotted line) and from H $\alpha$ (solid line, taken from Martin \& Kennicutt 2001) for the 9 galaxies in common with Martin \& Kennicutt (2001), having a threshold radius larger than $90^{\prime \prime}$ (so that our data resolve it). The radius is normalized by the "threshold radius" $R_{\text {threshold }}$ measured by Martin \& Kennicutt (2001). We show only a region around the threshold radius, marked by a vertical dashed line. Note that for the $\mathrm{H} \alpha$ profiles, the horizontal parts of the profiles beyond $R / R_{\text {threshold }} \sim 1$ are actually upper limits. The $\mathrm{H} \alpha$ profiles have a much higher resolution $\left(10^{\prime \prime}\right)$ than the UV + far-infrared data (resolution shown by the error bar in the central bottom part of each panel); however, we have many UV points beyond the threshold without any sign of a decrease in the UV for most galaxies. [See the electronic edition of the Supplement for a color version of this figure.]

The apparent absence of threshold may seem at odds with the figures presented in the Appendix, in which an abrupt drop of the UV surface brightness (or SFR surface density) is visible for a few galaxies. This is the case for instance of NGC 0891. However, for this galaxy, the UV drop is concomitant with a drop in the gas density and occurs at quite large radius $(35 \mathrm{kpc})$. The gas may be affected for various reasons at large radii (e.g., gravitational interactions, ram pressure), and obviously no star formation would occur when the gas reservoir was removed. Thus, while abrupt drops exist in the UV for some galaxies, they do not always correspond to a "threshold" of star formation. NGC 0925 presents a more interesting case where no UV is detected beyond about
$20 \mathrm{kpc}$, while $\mathrm{H}$ i is still observed. Even if this indicates for this galaxy a "UV threshold," our analysis includes galaxies with detected star formation at similar gas densities. Detailed studies of individual galaxies with high resolution may help to understand the peculiarities of system like this. This kind of analysis is beyond the scope of this paper.

Despite these questions on possible "UV thresholds," it is clear from Figure 7 that beyond the usual threshold radius, we frequently see UV radiation when $\mathrm{H} \alpha$ ceased to be steadily found. While many reasons for this are possible, including a variation in the initial mass function, the simplest explanation is that at some radius, star formation levels are so low that the number of 


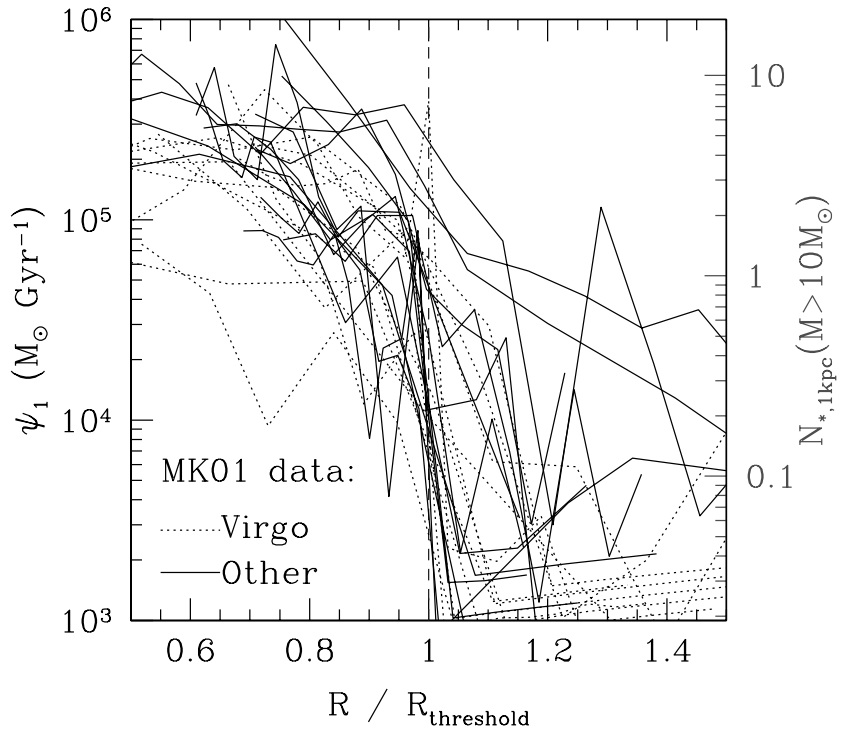

FIG. 8.- Profiles of the star formation rate surface density determined from the $\mathrm{H} \alpha$ profiles of Martin \& Kennicutt (2001). The left axis indicates the star formation rate integrated in $1 \mathrm{kpc}$ annular ellipses. The right axis shows the number ionizing stars within the same annular ellipses (see text for details). Dotted lines correspond to galaxies in Virgo. The radius is normalized by the "threshold radius" $R_{\text {threshold }}$ measured by Martin \& Kennicutt (2001). We show only a region around the threshold radius, marked by a vertical dashed line. Note that several points beyond $R / R_{\text {threshold }}$ (straight, almost horizontal lines) are actually upper limits. [See the electronic edition of the Supplement for a color version of this figure.]

high-mass (short-lived) ionizing stars visible at any time becomes vanishingly small, while slightly longer lived stars, emitting in the UV for a correspondingly longer time, are more frequent. If this interpretation is correct, the origin of the threshold of star formation, largely debated on theoretical grounds (Toomre 1964; Quirk 1972; Wang \& Silk 1994; Martin \& Kennicutt 2001; Wong $\&$ Blitz 2002; Boissier et al. 2003; Schaye 2004) on the basis of $\mathrm{H} \alpha$ observations would be simply the lack of ionizing stars.

To further test this idea, we took the $\mathrm{H} \alpha$ profiles of Martin \& Kennicutt (2001) around the threshold radius they defined. Since profiles are usually computed within annular ellipses, we converted them to star formation rate integrated within $1 \mathrm{kpc}$ wide annular ellipses (that we will call $\psi_{1}$ ), as shown in Figure 8. For galaxies at $17 \mathrm{Mpc}$ (distance of the Virgo galaxies, dotted lines), the resolution of $10^{\prime \prime}$ of Martin \& Kennicutt (2001) correspond to $0.8 \mathrm{kpc}$, thus $\psi_{1}$ is actually close to the quantity really involved in measurements.

This star formation rate $\psi_{1}$ at the threshold curiously shows very low scatter around $4 \times 10^{4} M_{\odot} \mathrm{Gyr}^{-1}$. The right axis of Figure 8 shows $\psi_{1}$ in the unit of number of massive stars present at any time within the $1 \mathrm{kpc}$ wide annular ellipse. To compute this number, we integrated the initial mass function (assumed to be a Salpeter function over the range 0.1 and $100 M_{\odot}$ ) above $10 M_{\odot}$ and assumed a lifetime for these stars $\left(10^{7} \mathrm{yr}\right)$. Our adopted limit of $10 M_{\odot}$ being conservative, our estimate should be an upper limit. Note that the prediction of the number of $\mathrm{O}$ stars using Starburst 99 (Leitherer et al. 1999) with solar metallicity and a Salpeter initial mass function between 1 and $100 M_{\odot}$ is very close to the numbers we obtained in that way. Introducing lower mass stars into the initial mass function would reduce this number; thus once again, our number is probably an upper limit to the number of ionizing stars.

From Figure 8 we conclude that the threshold of star formation found by Martin \& Kennicutt (2001) corresponds to the radius where the number of ionizing stars at any given time within $1 \mathrm{kpc}$ wide ellipses becomes lower than about 1 . At the position of the threshold, we would expect to find more than one star in only about $19 \%$ of the galaxies of the galaxies of Martin \& Kennicutt (2001). For three stars, the percentage drops to about $6 \%$. It is thus natural that this radius will be the one where we stop finding $\mathrm{H}$ II regions, not because of the absence of star formation, but because there is a diminishingly small chance of catching a massive star in our annuli, even with a normal initial mass function.

Although the threshold radius is defined by an abrupt drop in the $\mathrm{H} \alpha$ surface brightness profile, Martin \& Kennicutt (2001) themselves noticed that many galaxies exhibit a few $\mathrm{H}$ II regions well beyond this radius, where star formation does seem more stochastic (see also the $\mathrm{H}$ II regions found in extreme outer parts of disk galaxies by Ferguson et al. 1998). The presence of resolved young blue B stars in the outskirts of M31 (Cuillandre et al. 2001) also shows that some star formation occurs below the critical gas surface density. Similarly, Davidge (2003) has shown that intermediate-age stars are presents at large radii in M33 and NGC 2403, suggesting again that star formation has proceeded in the past beyond the current threshold radius. All these facts are totally consistent with our hypothesis that the threshold radius is only marking the last radius where $\mathrm{H}$ II regions are found in sufficient numbers to compute an $\mathrm{H} \alpha$ profile, and not the end of star formation.

This is certainly relevant to the phenomenon of extended UV (XUV) disks in which UV emission is observed at large radii (and associated with relatively recent star formation) but where $\mathrm{H} \alpha$ detections are rare (Gil de Paz et al. 2005; Thilker et al. 2005). The situation is, however, certainly not that simple since XUV disks are themselves not obvious extrapolations of the inner UV profiles. It will, of course, be interesting to compare our results with the relation between gas and star formation rate in XUV galaxies. We plan to start that analysis with the spectacular case of NGC 4625 (A. Gil de Paz et al. 2008, in preparation).

\section{CONCLUSION}

Using the galaxies of large angular size culled from the GALEX Atlas of Nearby Galaxies (Gil de Paz et al. 2007), we have compiled GALEX UV and IRAS IR images to compute profiles of attenuation and star formation in 43 galaxies. We find typically that UV attenuation decreases, from a few magnitudes of extinction in the centers of galaxies, to low values at larger radii. Combined with the metallicity gradients observed in those same galaxies, this gives rise to a attenuation-metallicity relationship, quantified in $\S 3.4$. No clear correlation is found between the attenuation and the gas surface density.

Using the attenuation to correct the FUV profiles for dust, we derive star formation rate profiles with which we test several modern expressions of the classical "Schmidt law." Our data overlap with those of Kennicutt (1998a) but extend to significantly lower gas densities, suggesting that the UV light in general traces and reveals lower levels of star formation than $\mathrm{H} \alpha$, in coincidence with the recent GALEX discoveries of UV extended (XUV) disks (e.g., Gil de Paz et al. 2005; Thilker et al. 2005). We suggest that the much-debated threshold of star formation might have been an observational selection effect caused by the extremely low number of ionizing stars expected to be found beyond the "threshold" radius.

Indeed, for low levels of star formation, the $\mathrm{H} \alpha$ radiation in one elliptical annuli seems stochastic when the number of massive stars in this annuli becomes close to $\sim 1$. The UV radiation coming from less massive and more long-lived stars (thus in 
larger number) does not present a break in its radial profile at the $\mathrm{H} \alpha$ threshold radius.

While these results are already interesting in their own right, it is obviously time to look to more galaxies, using Spitzer data in combination with GALEX. Spitzer will allow us to include many more galaxies in such studies owing to its better resolution than IRAS. Indeed, a joint analysis of GALEX and Spitzer data is already underway in a SINGS-GALEX collaboration. Note that the SINGS sample will only partially overlap with the one presented here as some of the closest galaxies were not included in SINGS.
GALEX (Galaxy Evolution Explorer) is a NASA Small Explorer, launched in 2003 April. We gratefully acknowledge NASA's support for construction, operation, and science analysis for the GALEX mission, developed in cooperation with the Centre National d'Études Spatiales of France and the Korean Ministry of Science and Technology. A. G. d. P. is partially financed by the Spanish Programa Nacional de Astronomía y Astrofísica under grant AYA2003-01676. We also thank the MAGPOP network for its support, and the referee for very constructive comments.

Facilities: GALEX

Note added in proof.- It has come to our attention that there was an error in the script producing Fig. 8. The vertical axis should be multiplied by 1000 . The main consequence of this correction is that the difference between the H $\alpha$ and UV profiles cannot be explained by a simple stochasticity effect alone. We will explore this question in more detail in a future paper.

\section{APPENDIX A}

\section{INDIVIDUAL PROFILES}

The left panels of Figure Set 9 show the profiles of UV attenuation, UV surface brightness (corrected for attenuation and inclination) equivalent to a star formation rate surface density, and gas surface density used in this work. The right panels show gaseous hydrogen surface densities $\left(\mathrm{H} \mathrm{I}, \mathrm{H}_{2}, \mathrm{H} \mathrm{I}+\mathrm{H}_{2}\right)$ versus the star formation rate surface density.

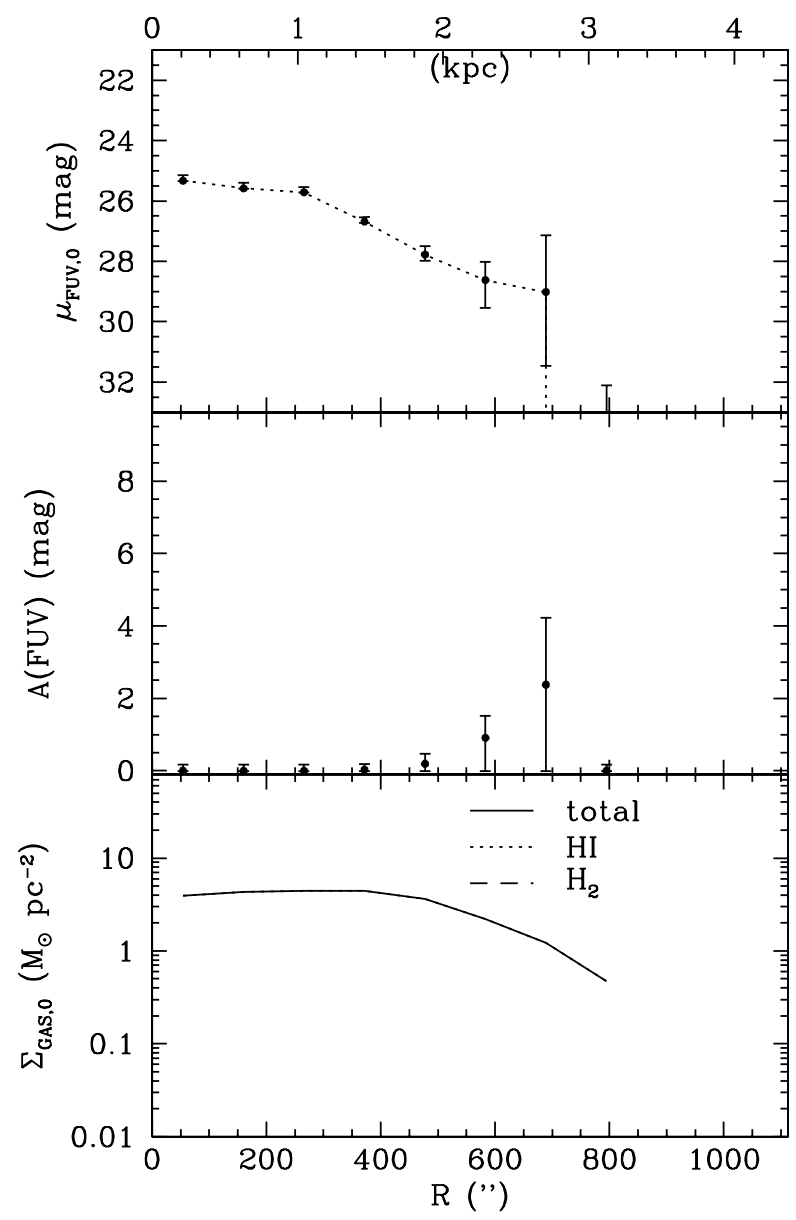

IC 1613

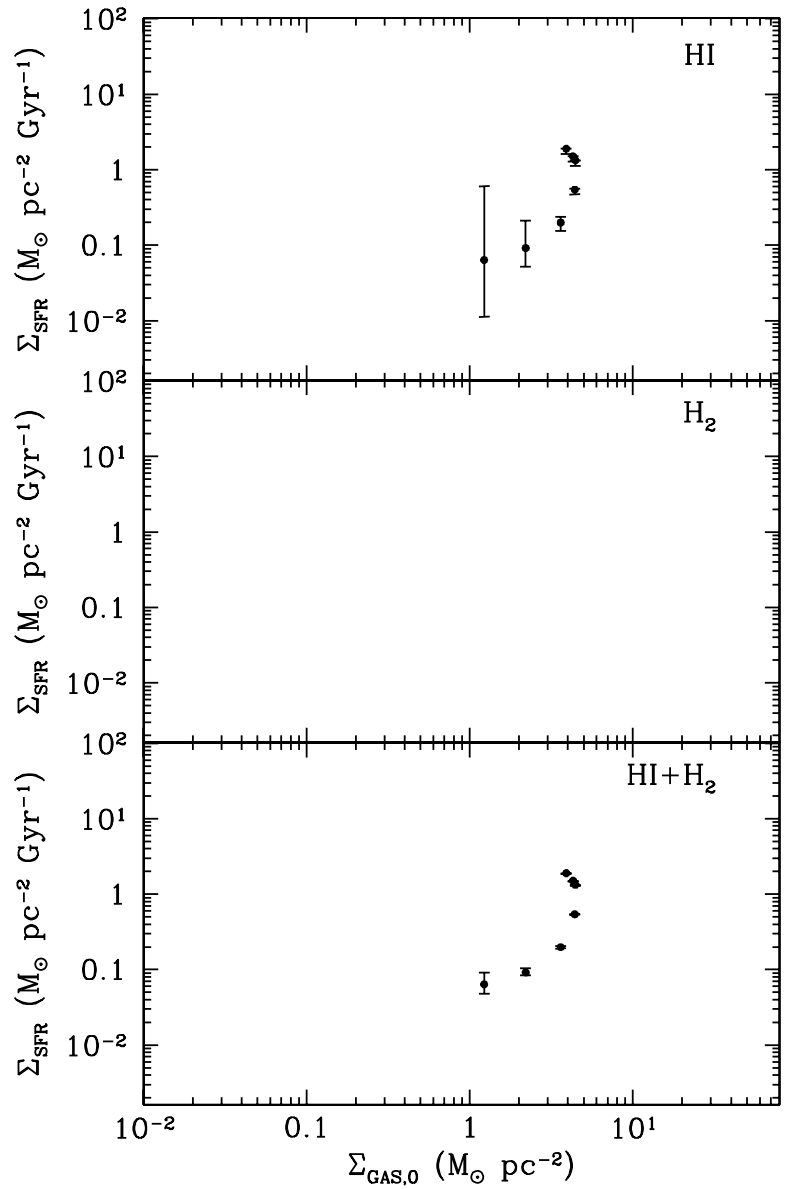

FIG. 9.- Radial profiles of the gas surface density (bottom left), FUV attenuation (middle left), and FUV surface brightness (corrected for attenuation and to a face-on inclination, top left) when available. This FUV surface brightness is directly proportional to the star formation rate surface density shown in the right panels as a function of the $\mathrm{H} \mathrm{I}, \mathrm{H}_{2}$, and the total gaseous hydrogen surface density along those profiles. Error bars in the left panels indicate how much the results would change if our sky determination in the UV or far-infrared was moved by $\pm 1 \sigma(\operatorname{see} \S 2)$. The name of the galaxy is indicated at the top. [See the electronic edition of the Supplement for Figs. 9.2-9.43.] 
Aumann, H. H., Fowler, J. W., \& Melnyk, M. 1990, AJ, 99, 1674

Bajaja, E., van der Burg, G., Faber, S. M., Gallagher, J. S., Knapp, G. R., \& Shane, W. W. 1984, A\&A, 141, 309

Bajaja, E., Wielebinski, R., Reuter, H.-P., Harnett, J. I., \& Hummel, E. 1995, A\&AS, 114, 147

Barnes, J. E. 2004, MNRAS, 350, 798

Begeman, K. G. 1987, Ph.D. thesis, Kapteyn Inst. 1989, A\&A, 223, 47

Bell, E. F. 2002, ApJ, 577, 150

Bell, E. F., Gordon, K. D., Kennicutt, R. C., \& Zaritsky, D. 2002, ApJ, 565, 994

Belley, J., \& Roy, J.-R. 1992, ApJS, 78, 61

Bersier, D., Blecha, A., Golay, M., \& Martinet, L. 1994, A\&A, 286, 37

Bohlin, R. C., Savage, B. D., \& Drake, J. F. 1978, ApJ, 224, 132

Boissier, S., Boselli, A., Buat, V., Donas, J., \& Milliard, B. 2004, A\&A, 424, 465

Boissier, S., \& Prantzos, N. 1999, MNRAS, 307, 857 -. 2000, MNRAS, 312, 398

Boissier, S., Prantzos, N., Boselli, A., \& Gavazzi, G. 2003, MNRAS, 346, 1215

Boissier, S., et al. 2005, ApJ, 619, L83

Boselli, A., Boissier, S., Cortese, L., Gil de Paz, A., Seibert, M., Madore, B. F., Buat, V., \& Martin, D. C. 2006, ApJ, 651, 811

Boselli, A., Gavazzi, G., Lequeux, J., Buat, V., Casoli, F., Dickey, J., \& Donas, J. 1995, A\&A, 300, L13

Boselli, A., Lequeux, J., \& Gavazzi, G. 2002, A\&A, 384, 33

Bosma, A. 1978, Ph.D. thesis, Groningen Univ. 1981, AJ, 86, 1791

Broeils, A. H., \& van Woerden, H. 1994, A\&AS, 107, 129

Braun, R. 1997, ApJ, 484, 637

Braun, R., Walterbos, R. A. M., Kennicutt, R. C., \& Tacconi, L. J. 1994, ApJ, 420, 558

Buat, V. 1989, A\&A, 220, 49

Buat, V., Boselli, A., Gavazzi, G., \& Bonfanti, C. 2002, A\&A, 383, 801

Buat, V., Deharveng, J. M., \& Donas, J. 1989, A\&A, 223, 42

Buat, V., \& Xu, C. 1996, A\&A, 306, 61

Buat, V., et al. 2005, ApJ, 619, L51

Burgarella, D., Buat, V., \& Iglesias-Páramo, J. 2005, MNRAS, 360, 1413

Calzetti, D., Kinney, A. L., \& Storchi-Bergmann, T. 1994, ApJ, 429, 582

Calzetti, D., et al. 2005, ApJ, 633, 871

Carignan, C., \& Puche, D. 1990a, AJ, 100, 394

1990b, AJ, 100, 641

Casertano, S., \& van Gorkom, J. H. 1991, AJ, 101, 1231

Cayatte, V., Kotanyi, C., Balkowski, C., \& van Gorkom, J. H. 1994, AJ, 107, 1003

Corbelli, E. 2003, MNRAS, 342, 199

Cortese, L., et al. 2006, ApJ, 637, 242

Cuillandre, J.-C., Lequeux, J., Allen, R. J., Mellier, Y., \& Bertin, E. 2001, ApJ, 554,190

Dale, D. A., Helou, G., Contursi, A., Silbermann, N. A., \& Kolhatkar, S. 2001, ApJ, 549, 215

Dame, T. M., Koper, E., Israel, F. P., \& Thaddeus, P. 1993, ApJ, 418, 730

Davidge, T. J. 2003, AJ, 125, 3046

Donas, J., Deharveng, J. M., Laget, M., Milliard, B., \& Huguenin, D. 1987, A\&A, 180, 12

Dopita, M. A., \& Ryder, S. D. 1994, ApJ, 430, 163

Elmegreen, B. G. 2002, ApJ, 577, 206

Ferguson, A. M. N., Wyse, R. F. G., Gallagher, J. S., \& Hunter, D. A. 1998, ApJ, 506, L19

Gavazzi, G., Zaccardo, A., Sanvito, G., Boselli, A., \& Bonfanti, C. 2004, A\&A, 417, 499

Gil de Paz, A., et al. 2005, ApJ, 627, L29 2007, ApJS, 173, 185

Hatton, S., Devriendt, J. E. G., Ninin, S., Bouchet, F. R., Guiderdoni, B., \& Vibert, D. 2003, MNRAS, 343, 75

Heckman, T., et al. 1995, ApJ, 452, 549

Heckman, T. M., Robert, C., Leitherer, C., Garnett, D. R., \& van der Rydt, F. 1998, ApJ, 503, 646

Heyer, M. H., Corbelli, E., Schneider, S. E., \& Young, J. S. 2004, ApJ, 602, 723

Holwerda, B. W., Gonzalez, R. A., Allen, R. J., \& van der Kruit, P. C. 2005a, AJ, 129, 1381 2005b, A\&A, 444, 101

Hunter, D. A., Elmegreen, B. G., \& van Woerden, H. 2001, ApJ, 556, 773

Jackson, D. C., Skillman, E. D., Cannon, J. M., \& Côté, S. 2004, AJ, 128, 1219

Jansen, R. A., Fabricant, D., Franx, M., \& Caldwell, N. 2000, ApJS, 126, 331

Kennicutt, R. C. 1989, ApJ, 344, 685

. 1998a, ApJ, 498, 541

1998b, ARA\&A, 36, 189

Kennicutt, R. C., Bresolin, F., \& Garnett, D. R. 2003a, ApJ, 591, 801
Kennicutt, R. C., et al. 2003b, PASP, 115, 928

Kenney, J. D. P., Scoville, N. Z., \& Wilson, C. D. 1991, ApJ, 366, 432

Kenney, J. D., \& Young, J. S. 1988, ApJS, 66, 261

Komugi, S., Sofue, Y., Nakanishi, H., Onodera, S., \& Egusa, F. 2005, PASJ, 57, 733

Kong, X., Charlot, S., Brinchmann, J., \& Fall, S. M. 2004, MNRAS, 349, 769

Lake, G., \& Skillman, E. D. 1989, AJ, 98, 1274

Lee, H., Grebel, E. K., \& Hodge, P. W. 2003, A\&A, 401, 141

Lee, H., McCall, M. L., Kingsburgh, R. L., Ross, R., \& Stevenson, C. C. 2003 , $\mathrm{AJ}, 125,146$

Leitherer, C., et al. 1999, ApJS, 123, 3

Lia, C., Portinari, L., \& Carraro, G. 2002, MNRAS, 330, 821

Madore, B. F. 1977, MNRAS, 178, 1

Martimbeau, N., Carignan, C., \& Roy, J.-R. 1994, AJ, 107, 543

Martin, C. L., \& Kennicutt, R. C. 2001, ApJ, 555, 301

Masegosa, J., Moles, M., \& del Olmo, A. 1991, A\&A, 249, 505

Meurer, G. R., Heckman, T. M., \& Calzetti, D. 1999, ApJ, 521, 64

Meurer, G. R., Heckman, T. M., Leitherer, C., Kinney, A., Robert, C., \& Garnett, D. R. 1995, AJ, 110, 2665

Morrissey, P., et al. 2005, ApJ, 619, L7

Mulder, P. S., \& van Driel, W. 1993, A\&A, 272, 63

Newton, K., \& Emerson, D. T. 1977, MNRAS, 181, 573

Nieten, C., Neininger, N., Guelin, M., Ungerechts, H., Lucas, R., Berkhuijsen, E. M., Beck, R., \& Wielebinski, R. 2006, A\&A, 453, 459

Ohnishi, T. 1975, Prog. Theor. Phys., 53, 1042

Ondrechen, M. P., van der Hulst, J. M., \& Hummel, E. 1989, ApJ, 342, 39

Paglione, T. A. D., et al. 2001, ApJS, 135, 183

Panuzzo, P., Bressan, A., Granato, G. L., Silva, L., \& Danese, L. 2003, A\&A, 409,99

Pisano, D. J., Wilcots, E. M., \& Elmegreen, B. G. 1998, AJ, 115, 975

Popescu, C. C., Misiriotis, A., Kylafis, N. D., Tuffs, R. J., \& Fischera, J. 2000, A\&A, 362, 138

Popescu, C. C., et al. 2005, ApJ, 619, L75

Prantzos, N., \& Aubert, O. 1995, A\&A, 302, 69

Puche, D., Carignan, C., \& Bosma, A. 1990, AJ, 100, 1468

Puche, D., Carignan, C., \& van Gorkom, J. H. 1991, AJ, 101, 456

Puche, D., Carignan, C., \& Wainscoat, R. J. 1991, AJ, 101, 447

Quirk, W. J. 1972, ApJ, 176, L9

Rand, R. J. 1994, A\&A, 285, 833

Regan, M. W., Thornley, M. D., Helfer, T. T., Sheth, K., Wong, T., Vogel, S. N., Blitz, L., \& Bock, D. C.-J. 2001, ApJ, 561, 218

Rice, W. 1993, AJ, 105, 67

Roy, J.-R., Belley, J., Dutil, Y., \& Martin, P. 1996, ApJ, 460, 284

Sakamoto, S., Handa, T., Sofue, Y., Honma, M., \& Sorai, K. 1997, ApJ, 475, 134

Sancisi, R., \& Allen, R. J. 1979, A\&A, 74, 73

Schaye, J. 2004, ApJ, 609, 667

Schiminovich, D., et al. 2005, ApJ, 619, L47

Schlegel, D. J., Finkbeiner, D. P., \& Davis, M. 1998, ApJ, 500, 525

Schmidt, M. 1959, ApJ, 129, 243

Seibert, M., et al. 2005, ApJ, 619, L55

Seigar, M. S. 2005, MNRAS, 361, L20

Shostak, G. S., \& van der Kruit, P. C. 1984, A\&A, 132, 20

Storchi-Bergmann, T., Wilson, A. S., \& Baldwin, J. A. 1996, ApJ, 460, 252

Sofue, Y. 1997, PASJ, 49, 17

Sorai, K., Nakai, N., Kuno, N., Nishiyama, K., \& Hasegawa, T. 2000, PASJ, 52,785

Thilker, D. A., et al. 2005, ApJ, 619, L79

2007, ApJS, 173, 572

Toomre, A. 1964, ApJ, 139, 1217

van Driel, W., Rots, A. H., \& van Woerden, H. 1988, A\&A, 204, 39

van Driel, W., Valentijn, E. A., Wesselius, P. R., \& Kussendrager, D. 1995, A\&A, 298, L41

van Zee, L., Salzer, J. J., Haynes, M. P., O’Donoghue, A. A., \& Balonek, T. J. 1998, AJ, 116, 2805

Vollmer, B., \& Beckert, T. 2002, A\&A, 382, 872

Wang, B., \& Silk, J. 1994, ApJ, 427, 759

Wevers, B. M. H. R., van der Kruit, P. C., \& Allen, R. J. 1986, A\&AS, 66, 505

Witt, A. N., \& Gordon, K. D. 2000, ApJ, 528, 799

Wong, T., \& Blitz, L. 2002, ApJ, 569, 157

Wyse, R. F. G., \& Silk, J. 1989, ApJ, 339, 700

Young, J. S., \& Scoville, N. 1982, ApJ, 260, L41

Young, J. S., et al. 1995, ApJS, 98, 219

Zaritsky, D., Kennicutt, R. C., \& Huchra, J. P. 1994, ApJ, 420, 87

Zhang, X., Wright, M., \& Alexander, P. 1993, ApJ, 418, 100 